\title{
Where do winds come from? A new theory on how water vapor condensation influences atmospheric pressure and dynamics
}

\author{
A. M. Makarieva ${ }^{1,2}$, V. G. Gorshkov ${ }^{1,2}$, D. Sheil ${ }^{3,4,5}$, A. D. Nobre ${ }^{6,7}$, and B.-L. Li ${ }^{2}$ \\ ${ }^{1}$ Theoretical Physics Division, Petersburg Nuclear Physics Institute, 188300, Gatchina, St. Petersburg, Russia \\ ${ }^{2}$ XIEG-UCR International Center for Arid Land Ecology, University of California, Riverside, CA 92521, USA \\ ${ }^{3}$ School of Environment, Science and Engineering, Southern Cross University, P.O. Box 157, Lismore, NSW 2480, Australia \\ ${ }^{4}$ Institute of Tropical Forest Conservation, Mbarara University of Science and Technology, Kabale, Uganda \\ ${ }^{5}$ Center for International Forestry Research, P.O. Box 0113 BOCBD, Bogor 16000, Indonesia \\ ${ }^{6}$ Centro de Ciência do Sistema Terrestre INPE, São José dos Campos SP 12227-010, Brazil \\ ${ }^{7}$ Instituto Nacional de Pesquisas da Amazônia, Manaus AM 69060-001, Brazil
}

Correspondence to: A. M. Makarieva (ammakarieva@gmail.com) and D. Sheil (douglassheil@itfc.org)

Received: 5 August 2010 - Published in Atmos. Chem. Phys. Discuss.: 15 October 2010

Revised: 29 April 2011 - Accepted: 3 December 2012 - Published: 25 January 2013

\begin{abstract}
Phase transitions of atmospheric water play a ubiquitous role in the Earth's climate system, but their direct impact on atmospheric dynamics has escaped wide attention. Here we examine and advance a theory as to how condensation influences atmospheric pressure through the mass removal of water from the gas phase with a simultaneous account of the latent heat release. Building from fundamental physical principles we show that condensation is associated with a decline in air pressure in the lower atmosphere. This decline occurs up to a certain height, which ranges from 3 to $4 \mathrm{~km}$ for surface temperatures from 10 to $30^{\circ} \mathrm{C}$. We then estimate the horizontal pressure differences associated with water vapor condensation and find that these are comparable in magnitude with the pressure differences driving observed circulation patterns. The water vapor delivered to the atmosphere via evaporation represents a store of potential energy available to accelerate air and thus drive winds. Our estimates suggest that the global mean power at which this potential energy is released by condensation is around one per cent of the global solar power - this is similar to the known stationary dissipative power of general atmospheric circulation. We conclude that condensation and evaporation merit attention as major, if previously overlooked, factors in driving atmospheric dynamics.
\end{abstract}

\section{Introduction}

Phase transitions of water are among the major physical processes that shape the Earth's climate. But such processes have not been well characterized. This shortfall is recognized both as a challenge and a prospect for advancing our understanding of atmospheric circulation (e.g., Lorenz, 1983; Schneider, 2006). In A History of Prevailing Ideas about the General Circulation of the Atmosphere Lorenz (1983) wrote:

\footnotetext{
"We may therefore pause and ask ourselves whether this step will be completed in the manner of the last three. Will the next decade see new observational data that will disprove our present ideas? It would be difficult to show that this cannot happen.

Our current knowledge of the role of the various phases of water in the atmosphere is somewhat incomplete: eventually it must encompass both thermodynamic and radiational effects. We do not fully understand the interconnections between the tropics, which contain the bulk of water, and the remaining latitudes ... Perhaps near the end of the 20th century we shall suddenly discover that we are beginning the fifth step."
}

Deluc (1812, p. 176) mentioned that conversion of water vapor to rain creates a kind of "airfree" space that may cause 
wind gusts. Lorenz (1967, Eq. 86), as well as several other authors after him (Trenberth et al., 1987; Trenberth, 1991; Gu and Qian, 1991; Ooyama, 2001; Schubert et al., 2001; Wacker and Herbert, 2003; Wacker et al., 2006), recognized that local pressure is reduced by precipitation and increased by evaporation. Qiu et al. (1993) noted that "the mass depletion due to precipitation tends to reduce surface pressure, which may in turn enhance the low-level moisture convergence and give a positive feedback to precipitation". Van den Dool and Saha (1993) labeled the effect as a physically distinct "water vapor forcing". Lackmann and Yablonsky (2004) investigated the precipitation mass sink for the case of Hurricane Lili (2002) and made an important observation that "the amount of atmospheric mass removed via precipitation exceeded that needed to explain the model sea level pressure decrease".

Although the pressure changes associated with evaporation and condensation have received some attention, the investigations have been limited: the effects remain poorly characterized in both theory and observations. Previous investigations focused on temporal pressure changes not spatial gradients. Even some very basic relationships remain subject to confusion. For example, there is doubt as to whether condensation leads to reduced or to increased atmospheric pressure (Pöschl, 2009, p. S12436). Opining that the status of the issue in the meteorological literature is unclear, Haynes (2009) suggested that to justify the claim of pressure reduction one would need to show that "the standard approaches (e.g., set out in textbooks such as "Thermodynamics of Atmospheres and Oceans" by Curry and Webster, 1999) imply a drop in pressure associated with condensation".

Here we aim to clarify and describe, building from basic and established physical principles, the pressure changes associated with condensation. We will argue that atmospheric water vapor represents a store of potential energy that becomes available to accelerate air as the vapor condenses. Evaporation, driven by the sun, continuously replenishes the store of this energy in the atmosphere.

The paper is structured as follows. In Sect. 2 we analyze the process of adiabatic condensation to show that it is always accompanied by a local decrease of air pressure. In Sect. 3 we evaluate the effects of water mass removal and lapse rate change upon condensation in a vertical air column in approximate hydrostatic equilibrium. In Sect. 4 we estimate the horizontal pressure gradients induced by water vapor condensation to show that these are sufficient enough to drive the major circulation patterns on Earth (Sect. 4.1). We examine why the key relationships have remained unknown until recently (Sects. 4.2 and 4.3). We evaluate the mean global power available from condensation to drive the general atmospheric circulation (Sect. 4.4). Finally, we discuss the interplay between evaporation and condensation and the essentially different implications of their physics for atmospheric dynamics (Sect. 4.5). In the concluding section we discuss the importance of condensation as compared to differential heating as the major driver of atmospheric circulation. Our theoretical investigations strongly suggest that the phase transitions of water vapor play a far greater role in driving atmospheric dynamics than is currently recognized.

\section{Condensation in a local air volume}

\subsection{Adiabatic condensation}

We will first show that adiabatic condensation is always accompanied by a decrease of air pressure in the local volume where it occurs. The first law of thermodynamics for moist air saturated with water vapor reads (e.g., Gill, 1982)

$\mathrm{d} Q=c_{\mathrm{V}} \mathrm{d} T+p \mathrm{~d} V+L \mathrm{~d} \gamma$,

$\gamma \equiv \frac{p_{\mathrm{v}}}{p} \ll 1, \frac{\mathrm{d} \gamma}{\gamma}=\frac{\mathrm{d} p_{\mathrm{v}}}{p_{\mathrm{v}}}-\frac{\mathrm{d} p}{p}$.

Here $p_{\mathrm{v}}$ is partial pressure of saturated water vapor, $p$ is air pressure, $T$ is absolute temperature, $Q\left(\mathrm{~J} \mathrm{~mol}^{-1}\right)$ is molar heat, $V\left(\mathrm{~m}^{3} \mathrm{~mol}^{-1}\right)$ is molar volume, $L \approx 45 \mathrm{~kJ} \mathrm{~mol}^{-1}$ is the molar heat of vaporization, $c_{\mathrm{V}}=\frac{5}{2} R$ is molar heat capacity of air at constant volume $\left(\mathrm{J} \mathrm{mol}^{-1} \mathrm{~K}^{-1}\right), R=$ $8.3 \mathrm{~J} \mathrm{~mol}^{-1} \mathrm{~K}^{-1}$ is the universal gas constant. The small value of $\gamma<0.1$ under terrestrial conditions allows us to neglect the influence made by the heat capacity of liquid water in Eq. (1).

The partial pressure of saturated water vapor obeys the Clausius-Clapeyron equation:

$\frac{\mathrm{d} p_{\mathrm{v}}}{p_{\mathrm{v}}}=\xi \frac{\mathrm{d} T}{T}, \xi \equiv \frac{L}{R T}$,

$p_{\mathrm{v}}(T)=p_{\mathrm{v} 0} \exp \left(\xi_{0}-\xi\right)$,

where $p_{\mathrm{v} 0}$ and $\xi_{0}$ correspond to some reference temperature $T_{0}$. Below we use $T_{0}=303 \mathrm{~K}$ and $p_{\mathrm{v} 0}=42 \mathrm{hPa}$ (Bolton, 1980) and neglect the dependence of $L$ on temperature.

We will also use the ideal gas law as the equation of state for atmospheric air:

$p V=R T$,

$\frac{\mathrm{d} p}{p}+\frac{\mathrm{d} V}{V}=\frac{\mathrm{d} T}{T}$.

Using Eq. (6) the first two terms in Eq. (1) can be written in the following form

$$
\begin{gathered}
c_{\mathrm{V}} \mathrm{d} T+p \mathrm{~d} V=\frac{R T}{\mu}\left(\frac{\mathrm{d} T}{T}-\mu \frac{\mathrm{d} p}{p}\right), \\
\mu \equiv \frac{R}{c_{p}}=\frac{2}{7}=0.29, c_{p}=c_{\mathrm{V}}+R .
\end{gathered}
$$


Writing $\mathrm{d} \gamma$ in Eq. (1) with use of Eqs. (2) and (3) as

$\frac{\mathrm{d} \gamma}{\gamma}=\xi \frac{\mathrm{d} T}{T}-\frac{\mathrm{d} p}{p}$

and using the definition of $\xi$ (Eq. 3) we arrive at the following form for the first law of thermodynamics (Eq. 1):

$\mathrm{d} Q=\frac{R T}{\mu}\left\{\frac{\mathrm{d} T}{T}\left(1+\mu \gamma \xi^{2}\right)-\mu \frac{\mathrm{d} p}{p}(1+\gamma \xi)\right\}$.

In adiabatic processes $\mathrm{d} Q=0$, and the expression in braces in Eq. (9) turns to zero, which implies:

$\frac{\mathrm{d} T}{T}=\frac{\mathrm{d} p}{p} \varphi(\gamma, \xi), \varphi(\gamma, \xi) \equiv \mu \frac{1+\gamma \xi}{1+\mu \gamma \xi^{2}} \equiv \varphi$.

Note that $\mu, \gamma$ and $\xi$ are all dimensionless; $\gamma$ and $\xi$ are variables and $\mu$ is a constant, $\varphi(0,0)=\mu$. This is a general dependence of temperature on pressure in an adiabatic atmospheric process that involves phase transitions of water vapor (evaporation or condensation), i.e. change of $\gamma$. At the same time $\gamma$ itself is a function of temperature as determined by Eq. (8):

$\frac{\mathrm{d} \gamma}{\gamma}=\xi \frac{\mathrm{d} T}{T}-\frac{\mathrm{d} p}{p}=\frac{\mathrm{d} T}{T} \frac{\xi \varphi-1}{\varphi}=(\xi \varphi-1) \frac{\mathrm{d} p}{p}$.

One can see from Eqs. (10) and (11) that the adiabatic phase transitions of water vapor are fully described by the relative change of either pressure $\mathrm{d} p / p$ or temperature $\mathrm{d} T / T$. For the temperature range relevant for Earth we have $\xi \equiv L / R T \approx 18$ so that

$\xi \mu-1 \approx 4.3$.

Noting that $\mu, \gamma, \xi$ are all positive, from Eqs. (10), (11) and (12) we obtain

$\xi \varphi-1=\xi \mu \frac{1+\gamma \xi}{1+\mu \gamma \xi^{2}}-1=\frac{\xi \mu-1}{1+\mu \gamma \xi^{2}}>0$.

Condensation of water vapor corresponds to a decrease of $\gamma, \mathrm{d} \gamma<0$. It follows unambiguously from Eqs. (11) and (13) that if $\mathrm{d} \gamma$ is negative, then $\mathrm{d} p$ and $\mathrm{d} T$ are negative too. This proves that water vapor condensation in any adiabatic process is necessarily accompanied by reduced air pressure.

\subsection{Adiabatic condensation cannot occur at constant volume}

Our previous result refutes the proposition that adiabatic condensation can lead to a pressure rise due to the release of latent heat (Pöschl, 2009, p. S12436). Next, we show that while such a pressure rise was implied by calculations assuming adiabatic condensation at constant volume, in fact such a process is prohibited by the laws of thermodynamics and thus cannot occur.
Using Eqs. (6), (10) and (8) we can express the relative change of molar volume $\mathrm{d} V / V$ in terms of $\mathrm{d} \gamma / \gamma$ :

$\frac{\mathrm{d} V}{V}=-\frac{1-\varphi}{\varphi \xi-1} \frac{\mathrm{d} \gamma}{\gamma}$

Putting $\mathrm{d} V=0$ in Eq. (14) we obtain

$\frac{(1-\varphi) \mathrm{d} \gamma}{(\xi \varphi-1) \gamma}=0$.

The denominator in Eq. (15) is greater than zero, see Eqs. (12) and (13). In the numerator we note from the definition of $\varphi$ (Eq. 10) that $1-\varphi=\frac{2 \gamma}{7+2 \gamma \xi^{2}}\left[\frac{5}{2 \gamma}+\xi(\xi-1)\right]$. The expression in square brackets lacks real roots:

$\frac{5}{2 \gamma}+\xi^{2}-\xi=0, \xi=\frac{1}{2}\left(1 \pm i \sqrt{\frac{10-\gamma}{\gamma}}\right), \gamma \leq 1$.

In consequence, Eq. (15) has a single solution $\mathrm{d} \gamma=0$. This proves that condensation cannot occur adiabatically at constant volume.

\subsection{Non-adiabatic condensation}

To conclude this section, we show that for any process where entropy increases, $\mathrm{d} S=\mathrm{d} Q / T>0$, water vapor condensation $(\mathrm{d} \gamma<0)$ is accompanied by drop of air pressure (i.e., $\mathrm{d} p<0)$. We write the first law of thermodynamics Eqs. (9) and (11) as

$\frac{\mathrm{d} S}{R} \frac{\mu}{1+\mu \gamma \xi^{2}}=\frac{\mathrm{d} T}{T}-\varphi \frac{\mathrm{d} p}{p}, \frac{\mathrm{d} T}{T}=\frac{1}{\xi}\left(\frac{\mathrm{d} \gamma}{\gamma}+\frac{\mathrm{d} p}{p}\right)$.

Excluding $\mathrm{d} T / T$ from Eq. (17) we obtain

$\frac{\mathrm{d} p}{p}(\xi \varphi-1)=\frac{\mathrm{d} \gamma}{\gamma}-\xi \frac{\mu}{1+\mu \gamma \xi} \frac{\mathrm{d} S}{R}$.

The term in round brackets in Eq. (18) is positive, see Eq. (13), the multiplier at $\mathrm{d} S$ is also positive. Therefore, when condensation occurs, i.e., when $\mathrm{d} \gamma / \gamma<0$, and $\mathrm{d} S>0$, the lefthand side of Eq. (18) is negative. This means that $\mathrm{d} p / p<0$, i.e., air pressure decreases.

Condensation can be accompanied by a pressure increase only if $\mathrm{d} S<0$. This requires that work is performed on the gas such as occurs if it is isothermally compressed. (We note too, that if pure saturated water vapor is isothermally compressed condensation occurs, but the Clausius-Clapeyron equation (Eq. 3) shows that the vapor pressure remains unchanged being purely a function of temperature.)

\section{Adiabatic condensation in the gravitational field}

\subsection{Difference in the effects of mass removal and temperature change on gas pressure in hydrostatic equilibrium}

We have shown that adiabatic condensation in any local volume is always accompanied by a drop of air pressure. We 
will now explore the consequences of condensation for the vertical air column.

Most circulation patterns on Earth are much wider than they are high, with the ratio height/length being in the order of $10^{-2}$ for hurricanes and down to $10^{-3}$ and below in larger regional circulations. As a consequence of mass balance, vertical velocity is smaller than horizontal velocities by a similar ratio. Accordingly, the local pressure imbalances and resulting atmospheric accelerations are much smaller in the vertical orientation than in the horizontal plane, the result being an atmosphere in approximate hydrostatic equilibrium (Gill, 1982). Air pressure then conforms to the equation

$-\frac{\mathrm{d} p}{\mathrm{~d} z}=\rho g, \quad p(0) \equiv p_{\mathrm{s}}=g \int_{0}^{\infty} \rho(z) \mathrm{d} z$.

Applying the ideal gas equation of state (Eq. 5) we have from Eq. (19)

$\frac{\mathrm{d} p}{\mathrm{~d} z}=-\frac{p}{h}, h \equiv \frac{R T}{M g}$.

This solves as

$p(z)=p_{\mathrm{s}} \exp \left\{-\int_{0}^{z} \frac{\mathrm{d} z^{\prime}}{h\left(z^{\prime}\right)}\right\}$.

Here $M$ is air molar mass $\left(\mathrm{kg} \mathrm{mol}^{-1}\right)$, which, as well as temperature $T(z)$, in the general case also depends on $z$.

The value of $p_{\mathrm{s}}$ (Eq. 19), air pressure at the surface, appears as the constant of integration after Eq. (19) is integrated over $z$. It is equal to the weight of air molecules in the atmospheric column. It is important to bear in mind that $p_{\mathrm{s}}$ does not depend on temperature, but only on the amount of gas molecules in the column. It follows from this observation that any reduction of gas content in the column reduces surface pressure.

Latent heat released when water condenses means that more energy has to be removed from a given volume of saturated air for a similar decline in temperature when compared to dry air. This is why the moist adiabatic lapse rate is smaller than the dry adiabatic lapse rate. Accordingly, given one and the same surface temperature $T_{\mathrm{S}}$ in a column with rising air, the temperature at some distance above the surface will be on average higher in a column of moist saturated air than in a dry one.

However, this does not mean that at a given height air pressure in the warmer column is greater than air pressure in the colder column (cf. Meesters et al., 2009; Makarieva and Gorshkov, 2009c), because air pressure $p(z)$ (Eq. 21) depends on two parameters, temperature $T(z)$ and surface air pressure (i.e., the total amount of air in the column). If the total amount of air in the warmer column is smaller than in the colder column, air pressure in the surface layer will be lower in the warmer column despite its higher temperature.
In the following we estimate the cumulative effect of gas content and lapse rate changes upon condensation.

\subsection{Moist adiabatic temperature profile}

Relative water vapor content (Eq. 2) and temperature $T$ depend on height $z$. From Eqs. (10), (11) and (20) we have

$$
\begin{aligned}
& -\frac{\mathrm{d} T}{\mathrm{~d} z} \equiv \Gamma=\varphi \frac{T}{h}, \quad \varphi \equiv \mu \frac{1+\gamma \xi}{1+\gamma \mu \xi^{2}}, \\
& -\frac{1}{\gamma} \frac{\mathrm{d} \gamma}{\mathrm{d} z}=\frac{\xi \varphi-1}{h} \equiv \frac{\xi \mu-1}{1+\mu \gamma \xi^{2}} \frac{1}{h} .
\end{aligned}
$$

Equation (22) represents the well-known formula for a moist adiabatic gradient as given in Glickman (2000) for small $\gamma<0.1$. At $\gamma=0$ we have $\varphi(\gamma, \xi)=\mu$ and $\Gamma_{\mathrm{d}}=M_{\mathrm{d}} g / c_{p}=$ $9.8 \mathrm{~K} \mathrm{~km}^{-1}$, which is the dry adiabatic lapse rate that is independent of height $z, M_{\mathrm{d}}=29 \mathrm{~g} \mathrm{~mol}^{-1}$. For moist saturated air the change of temperature $T$ and relative partial pressure $\gamma$ of water vapor with height is determined by the system of differential equations (Eqs. 22, 23).

Differentiating both parts of the Clapeyron-Clausius equation (Eq. 3) over $z$ we have, see Eq. (22):

$$
\begin{aligned}
& \frac{\mathrm{d} p_{\mathrm{v}}}{\mathrm{d} z}=-\frac{p_{\mathrm{v}}}{h_{\mathrm{v}}}, h_{\mathrm{v}} \equiv \frac{R T^{2}}{L \Gamma}=\frac{T}{\xi \Gamma}=\frac{h}{\xi \varphi}, \\
& p_{\mathrm{v}}(z)=p_{\mathrm{vs}} \exp \left\{-\int_{0}^{z} \frac{\mathrm{d} z^{\prime}}{h_{\mathrm{v}}}\right\}, p_{\mathrm{vs}} \equiv p_{\mathrm{v}}(0) .
\end{aligned}
$$

The value of $h_{\mathrm{v}}$ represents a fundamental scale height for the vertical distribution of saturated water vapor. At $T_{\mathrm{s}}=300 \mathrm{~K}$ this height $h_{\mathrm{v}}$ is approximately $4.5 \mathrm{~km}$.

Differentiating both parts of Eq. (2) over $z$ with use of Eqs. (20) and (24) and noticing that $h_{\mathrm{v}}=h /(\xi \varphi)$ we have

$$
\begin{aligned}
-\frac{1}{\gamma} \frac{\mathrm{d} \gamma}{\mathrm{d} z} & =\frac{1}{p_{\mathrm{v}}} \frac{\mathrm{d} p_{\mathrm{v}}}{\mathrm{d} z}-\frac{1}{p} \frac{\mathrm{d} p}{\mathrm{~d} z}=\frac{1}{h_{\mathrm{v}}}-\frac{1}{h} \equiv \frac{1}{h_{\gamma}}, \\
h_{\gamma} & \equiv \frac{h_{\mathrm{v}} h}{h-h_{\mathrm{v}}} .
\end{aligned}
$$

This equation is equivalent to Eq. (23) when Eqs. (22) and (24) are taken into account. Height $h_{\gamma}$ represents the vertical scale of the condensation process. Height scales $h_{\mathrm{v}}$ (Eq. 24) and $h_{\gamma}$ (Eq. 25) depend on $\varphi(\gamma, \xi)$ (Eq. 22) and, consequently, on $\gamma$. At $T_{\mathrm{s}}=300 \mathrm{~K}$ height $h_{\gamma} \approx 9 \mathrm{~km}$, in close proximity to the water vapor scale height described by Mapes (2001).

\subsection{Pressure profiles in moist versus dry air columns}

We start by considering two static vertically isothermal atmospheric columns of unit area, A and B, with temperature $T(z)=T_{\mathrm{s}}$ independent of height. Column A contains moist air with water vapor saturated at the surface, column B contains dry air only. Surface temperatures and surface pressures 


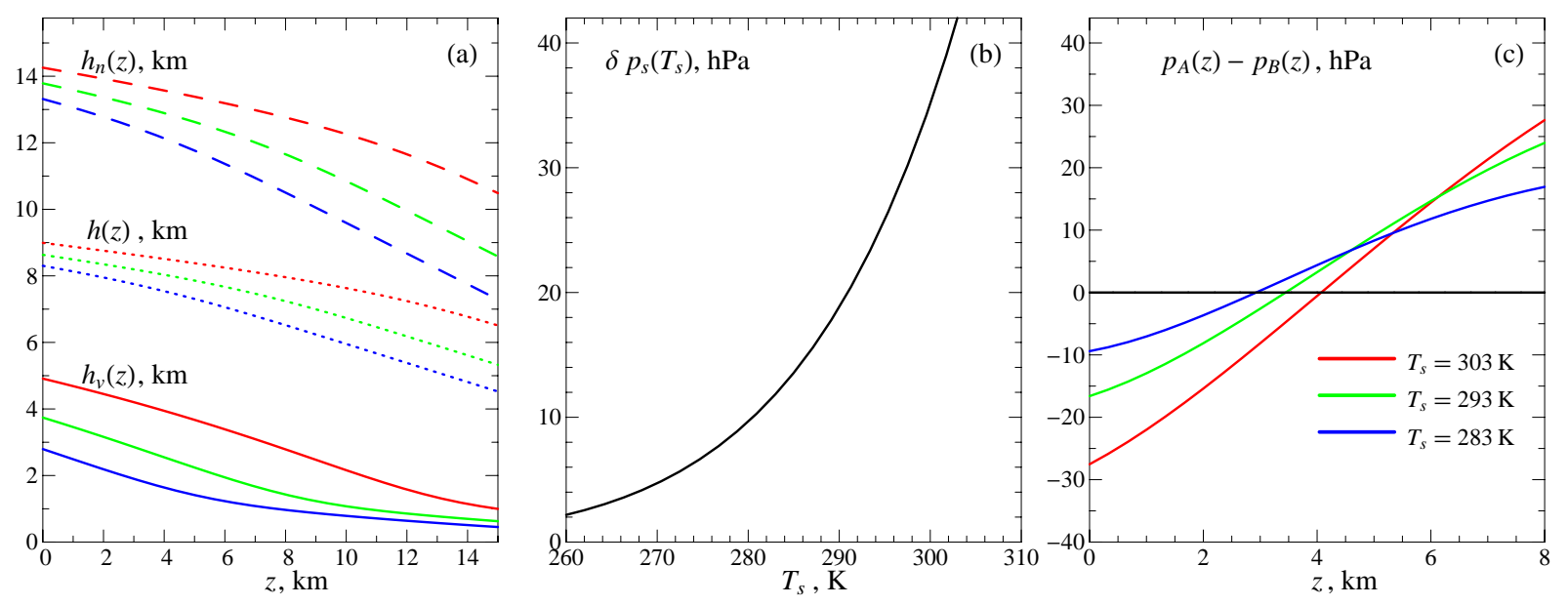

Fig. 1. (a) Scale height of saturated water vapor $h_{\mathrm{v}}(z)$ (Eq. 24), hydrostatic scale height of water vapor $h_{n}(z)$ (Eq. 26), and scale height of moist air $h(z)$ (Eq. 20) in the column with moist adiabatic lapse rate (Eq. 22) for three values of surface temperature $T_{\mathrm{s}}$; (b) condensationinduced drop of air pressure at the surface (Eq. 27) as dependent on surface temperature $T_{\mathrm{s}}$; (c) pressure difference versus altitude $z$ between atmospheric columns A and B with moist and dry adiabatic lapse rates, Eqs. (30) and (31), respectively, for three values of surface temperature $T_{\mathrm{S}}$. Height $z_{c}$ at which $p_{\mathrm{A}}\left(z_{c}\right)-p_{\mathrm{B}}\left(z_{c}\right)=0$ is $2.9,3.4$ and $4.1 \mathrm{~km}$ for 283, 293 and $303 \mathrm{~K}$, respectively. Due to condensation, at altitudes below $z_{c}$ the air pressure is lower in column A despite it being warmer than column B.

in the two columns are equal. In static air Eq. (19) is exact and applies to each component of the gas mixture as well as to the mixture as a whole. At equal surface pressures, the total air mass and air weight are therefore the same in both columns. Water vapor in column A is saturated at the surface (i.e., at $z=0$ ) but non-saturated above it (at $z>0$ ). The saturated partial pressure of water vapor at the surface $p_{\mathrm{v}}\left(T_{\mathrm{S}}\right)$ (Eq. 4) is determined by surface temperature and, as it is in hydrostatic equilibrium, equals the weight of water vapor in the static column.

We now introduce a non-zero lapse rate to both columns: the moist adiabatic $\Gamma$ (Eq. 22) to column A and the dry adiabatic $\Gamma_{\mathrm{d}}$ in column B. (Now the columns cannot be static: the adiabatic lapse rates are maintained by the adiabatically ascending air.) Due to the decrease of temperature with height, some water vapor in column A undergoes condensation. Water vapor becomes saturated everywhere in the column (i.e., at $z \geq 0$ ), with pressure $p_{\mathrm{v}}(z)$ following Eq. (24) and density $\rho_{\mathrm{v}}=p_{\mathrm{v}} M_{\mathrm{v}} /(R T) \equiv p_{\mathrm{v}} /\left(g h_{n}\right)$ following

$$
\begin{aligned}
& \rho_{\mathrm{v}}(z)=\rho_{\mathrm{v}}\left(T_{\mathrm{S}}\right) \frac{h_{n \mathrm{~s}}}{h_{n}(z)} \exp \left\{-\int_{0}^{z} \frac{\mathrm{d} z^{\prime}}{h_{\mathrm{v}}\left(z^{\prime}\right)}\right\}, \\
& \rho_{\mathrm{v}}\left(T_{\mathrm{S}}\right) \equiv \frac{p_{\mathrm{v}}\left(T_{\mathrm{S}}\right)}{g h_{n}\left(T_{\mathrm{S}}\right)}, h_{n} \equiv \frac{R T(z)}{M_{\mathrm{v}} g}, T(z)=T_{\mathrm{S}}-\Gamma z .
\end{aligned}
$$

Here $h_{n}(z)$ is the scale height of the hydrostatic distribution of water vapor in the isothermal atmosphere with $T(z)=T_{\mathrm{s}}$.

The change in pressure $\delta p_{\mathrm{s}}$ in column $\mathrm{A}$ due to water vapor condensation is equal to the difference between the initial weight of water vapor $p_{\mathrm{v}}\left(T_{\mathrm{S}}\right)$ and the weight of saturated water vapor:

$$
\begin{aligned}
\delta p_{\mathrm{s}} & =p_{\mathrm{v}}\left(T_{\mathrm{s}}\right)-g \int_{0}^{\infty} \rho_{\mathrm{v}}(z) \mathrm{d} z \leq p_{\mathrm{v}}\left(T_{\mathrm{s}}\right)-\rho_{\mathrm{v}}\left(T_{\mathrm{s}}\right) g h_{\mathrm{v}}\left(T_{\mathrm{s}}\right) \\
& =p_{\mathrm{v}}\left(T_{\mathrm{s}}\right)\left(1-\frac{h_{\mathrm{vs}}}{h_{n \mathrm{~s}}}\right)=p_{\mathrm{v}}\left(T_{\mathrm{s}}\right)\left(1-\frac{M_{\mathrm{v}} g T_{\mathrm{s}}}{L \Gamma_{\mathrm{s}}}\right) .
\end{aligned}
$$

The inequality in Eq. (27) represents a conservative estimate of $\delta p_{\mathrm{s}}$ due to the approximation $h_{\mathrm{v}}(z)=h_{\mathrm{v}}\left(T_{\mathrm{s}}\right)$ made while integrating $\rho_{\mathrm{v}}(z)(26)$. As far as $h_{\mathrm{v}}(z)$ declines with height more rapidly than $h_{n}(z)$, Fig. 1a, the exact magnitude of this integral is smaller, while the value of $\delta p_{\mathrm{s}}$ is larger. The physical meaning of estimate (Eq. 27) consists in the fact that the drop of temperature with height compresses the water vapor distribution $h_{n \mathrm{~s}} / h_{\mathrm{vs}}$-fold compared to the hydrostatic distribution (Makarieva and Gorshkov, 2007, 2009a; Gorshkov et al., 2012).

The value of $\delta p_{\mathrm{s}}$ (Eq. 27) was calculated as the difference between the weight per unit surface area of vapor in the isothermal hydrostatic column and the weight of water vapor that condensed when a moist adiabatic lapse rate was applied. This derivation can also be understood in terms of the variable conventionally called the adiabatic liquid water content (e.g., Curry and Webster, 1999, Eq. 6.41). We can represent the total mixing ratio of moisture (by mass) as $q_{\mathrm{t}} \equiv$ $q_{\mathrm{v}}+q_{1}=\left(\rho_{\mathrm{v}}+\rho_{1}\right) / \rho$, where $\rho_{\mathrm{v}}$ is the mass of vapor and $\rho_{\mathrm{l}}$ is the mass of liquid water per unit air volume; $q_{\mathrm{t}} \ll 1$. The total adiabatic liquid water content in the column equals the integral of $q_{\mathrm{l}} \rho$ over $z$ at constant $q_{\mathrm{t}}, q_{1} \rho=q_{\mathrm{t}} \rho-q_{\mathrm{v}} \rho=q_{\mathrm{t}} \rho-\rho_{\mathrm{v}}$. The value of $\delta p_{\mathrm{s}}$ (Eq. 27) is equal to this integral (mass per unit area) multiplied by the gravitational acceleration (giving weight per unit area): 
$\delta p_{\mathrm{s}}=g \int_{0}^{\infty} q_{\mathrm{l}} \rho \mathrm{d} z=g\left(\int_{0}^{\infty} q_{\mathrm{t}} \rho \mathrm{d} z-\int_{0}^{\infty} \rho_{\mathrm{v}} \mathrm{d} z\right)$.

The first integral in braces gives the mass of vapor in the considered atmospheric column if water vapor were a noncondensable gas, $q_{\mathrm{v}}=q_{\mathrm{t}}=$ const. This term is analagous to the first term, $p_{\mathrm{v}}\left(T_{\mathrm{S}}\right)$, in the right-hand side of Eq. (27), where a static isothermal column was considered. The second term is identical to the second term, $g \int_{0}^{\infty} \rho_{\mathrm{v}} \mathrm{d} z$, in Eq. (27).

Using the definition of $h_{\mathrm{v}}\left(T_{\mathrm{S}}\right)$ (Eq. 24), $h_{n}\left(T_{\mathrm{s}}\right)$ (Eq. 26) and recalling that $M_{\mathrm{v}} / M_{\mathrm{d}}=0.62$ and $p_{\mathrm{v}}\left(T_{\mathrm{s}}\right)=\gamma_{\mathrm{s}} p_{\mathrm{s}}$, see Eq. (4), we obtain the following expression for the $\delta p_{\mathrm{s}}$ estimate (Eq. 27), Fig. 1b:

$$
\frac{\delta p_{\mathrm{s}}}{p_{\mathrm{s}}} \approx \gamma_{\mathrm{s}}\left(1-0.62 \frac{1+\gamma_{\mathrm{s}} \mu \xi_{\mathrm{s}}^{2}}{\mu \xi_{\mathrm{s}}+\gamma_{\mathrm{s}} \mu \xi_{\mathrm{s}}^{2}}\right) \text {. }
$$

Note that $\delta p_{\mathrm{s}} / p_{\mathrm{s}}$ is proportional to $\gamma_{\mathrm{s}}$ and increases exponentially with the rise of temperature.

After an approximate hydrostatic equilibrium is established, the vertical pressure profiles for columns A and B become, cf. Eq. (21):

$$
\begin{aligned}
& p_{\mathrm{A}}(z)=p_{\mathrm{s}}\left(1-\frac{\delta p_{\mathrm{s}}}{p_{\mathrm{s}}}\right) \exp \left\{-\int_{0}^{z} \frac{\mathrm{d} z^{\prime}}{h_{\mathrm{A}}\left(z^{\prime}\right)}\right\}, h_{\mathrm{A}} \equiv \frac{R T}{M g} \\
& p_{\mathrm{B}}(z)=p_{\mathrm{s}} \exp \left\{-\int_{0}^{z} \frac{\mathrm{d} z^{\prime}}{h_{\mathrm{B}}\left(z^{\prime}\right)}\right\}, h_{\mathrm{B}} \equiv \frac{R T_{\mathrm{d}}}{M_{\mathrm{d} g}} .
\end{aligned}
$$

Here $M(z)=M_{\mathrm{d}}(1-\gamma)+M_{\mathrm{v}} \gamma ; \gamma \equiv p_{\mathrm{v}}(z) / p_{\mathrm{A}}(z)$ and $T(z)$ obey Eqs. (22) and (23), $T_{\mathrm{d}}(z) \equiv T_{\mathrm{s}}-\Gamma_{\mathrm{d}} z$.

In Fig. 1c the difference $p_{\mathrm{A}}(z)-p_{\mathrm{B}}(z)$ is plotted for three surface temperatures, $T_{\mathrm{s}}=10^{\circ} \mathrm{C}, 20^{\circ} \mathrm{C}$ and $30^{\circ} \mathrm{C}$. In all three cases condensation has resulted in a lower air pressure in column A compared to column B everywhere below $z_{c} \approx 2.9,3.4$ and $4.1 \mathrm{~km}$, respectively. It is only above that height that the difference in lapse rates makes pressure in the moist column higher than in the dry column.

\subsection{Comparing forces due to condensation and buoyancy}

Fig. 1c describes the relative contributions of latent heat release and the condensation vapor sink to the horizontal pressure differences. This result can also be illustrated by comparing the vertical forces associated with phase transitions of water vapor.

The buoyant force acting per unit moist air volume can be written as

$$
\begin{aligned}
& f_{\mathrm{B}}=\rho_{\mathrm{p}} g\left(\frac{\rho}{\rho_{\mathrm{p}}}-1\right)= \\
& \rho_{\mathrm{p}} g\left(\frac{T(z)}{T_{\mathrm{d}}(z)} \frac{1}{1-\left(M_{\mathrm{v}} / M_{\mathrm{d}}\right) \gamma(z)}-1\right) .
\end{aligned}
$$

Here $\rho_{\mathrm{p}}$ is the density of the air moist air parcel that ascends in the environment with density $\rho$. (When $f_{\mathrm{B}}$ is taken per unit mass by dividing by density $\rho_{\mathrm{p}}$ and integrated over $z$, one obtains the convective available potential energy (CAPE) (Glickman, 2000), which represents work performed by the buoyant force on the rising air parcel. As work of the buoyant force on the air parcel that is descending dry adiabatically is usually negative, total energy available for a buoyancyinduced circulation can be close to zero even at large positive CAPE (Gorshkov et al., 2012).)

Figure 2a shows the buoyant force acting on an air volume from column A that rises moist adiabatically in the dry adiabatic environment of column B: $\rho_{\mathrm{p}}=p_{\mathrm{B}}(z) M / R T$, $\rho=p_{\mathrm{B}}(z) M_{\mathrm{d}} / R T_{\mathrm{d}}$. Here $p_{\mathrm{B}}$ is given by Eq. (31), $T_{\mathrm{d}}$ follows the dry adiabatic profile $T_{\mathrm{d}}(z)=T_{\mathrm{s}}-\Gamma_{\mathrm{d}} z$, where $\Gamma_{\mathrm{d}}=$ $9.8 \mathrm{~K} \mathrm{~km}^{-1}$, while temperature $T(z)$ and molar mass $M(z)=$ $M_{\mathrm{d}}\left[1-\left(M_{\mathrm{v}} / M_{\mathrm{d}}\right) \gamma(z)\right]$ of the rising air satisfy Eqs. (22)(23). The positive value of the buoyant force at the surface is due to the lower molar density of the moist versus dry air.

The same figure shows the condensation pressure gradient force that acts in the column where moist saturated air ascends adiabatically:

$$
f_{\mathrm{C}}=\frac{p_{\mathrm{v}}}{p} \frac{\partial p}{\partial z}-\frac{\partial p_{\mathrm{v}}}{\partial z}=-p \frac{\partial \gamma}{\partial z} \text {. }
$$

Here $p$ and $\gamma$ conform to Eqs. (22)-(23).

As Fig. 2a shows, the two forces have different spatial localization. The condensation force has a maximum in the lower atmosphere where the amount of vapor is maximized. The buoyant force grows with height following the accumulating difference between the moist adiabatic and dry adiabatic temperatures. At $T_{\mathrm{s}}=300 \mathrm{~km}$ at $z=8 \mathrm{~km}$ the difference theoretically amounts to over $50 \mathrm{~K}$.

The buoyant force estimated in Fig. 2 represents a theoretical upper limit that assumes no heat transfer between the ascending air and its environment. Maximum temperature differences observed in the horizontal direction in real weather systems are typically much smaller than $50 \mathrm{~K}$ at any height. Indeed, even in the warm-core tropical storms - i.e., in intense precipitation events - the horizontal temperature difference between the core and the external environment rarely exceeds a few degrees Kelvin (e.g., Knaff et al., 2000). In Fig. $2 b$ the same forces are plotted, but for the buoyant force estimated for an environment having a mean tropospheric lapse rate of $6.5 \mathrm{~K} \mathrm{~km}^{-1}$ (rather than the dry adiabatic lapse rate $9.8 \mathrm{~K} \mathrm{~km}^{-1}$ ). As Fig. $2 \mathrm{~b}$ shows, the magnitude of the buoyant force drops rapidly with diminishing differences in temperature. Convective available potential energy associated with the buoyant force shown in Fig. 2a is $\int_{0}^{8 \mathrm{~km}}\left(f_{\mathrm{B}} / \rho_{\mathrm{p}}\right) \mathrm{d} z=8.5 \times 10^{3} \mathrm{~J} \mathrm{~kg}^{-1}$. This figure is several times higher than the typical values calculated from the lapse rate soundings of the atmospheric column below $12 \mathrm{~km}$ height in the most intense convection events like thunderstorms and tornadoes (e.g., Thompson et al., 2003; Kis and Straka, 2010). Furthermore, in a recent comparison 

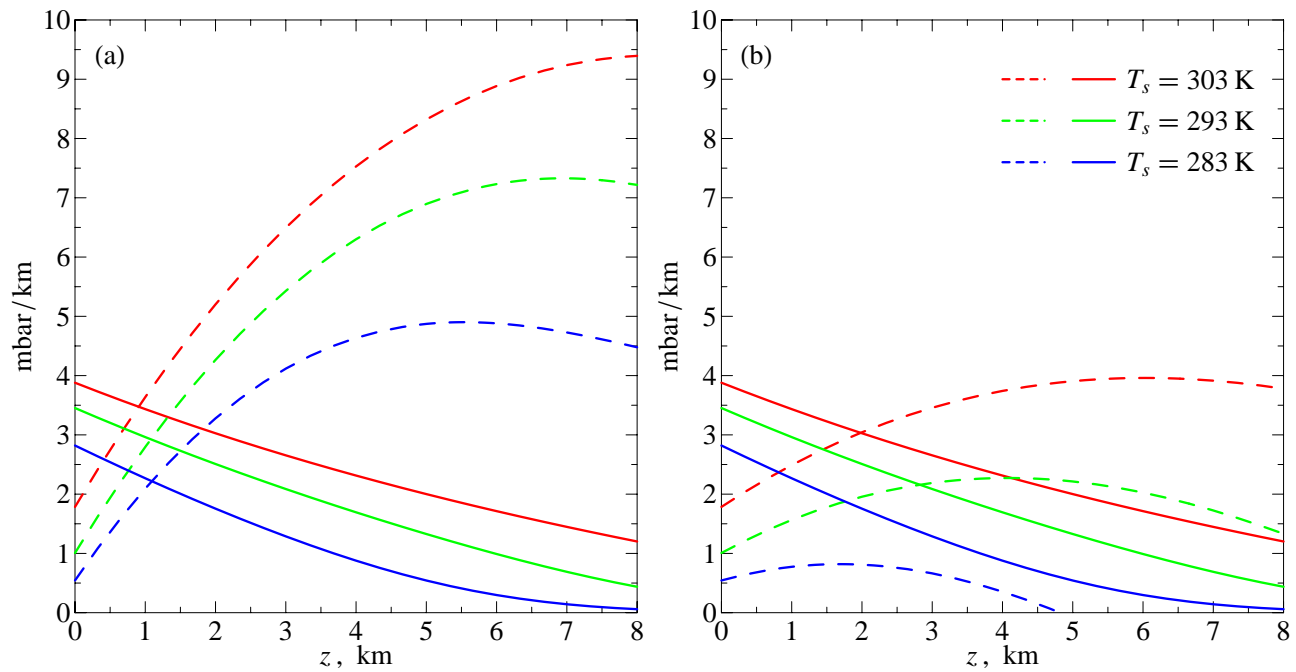

Fig. 2. Condensation force $f_{\mathrm{C}}$ (solid curves) and buoyant force $f_{\mathrm{B}}$ (dashed) acting at height $z$ on a moist air volume ascending in an environment with dry adiabatic lapse rate $9.8 \mathrm{~K} \mathrm{~km}^{-1}$ (a) and mean tropospheric lapse rate $6.5 \mathrm{~K} \mathrm{~km}^{-1}$ (b) for different values of surface temperature $T_{\mathrm{s}}$.

of nocturnal and diurnal tornadoes (Kis and Straka, 2010) it was found that significant tornadoes can form at both large and very small CAPE values, pointing to the importance of different mechanisms for the generation of intense circulation systems.

The key message from Fig. 2 is that the condensation force remains comparable in magnitude to the buoyant force even when the latter is allowed (for the sake of argument) to take unrealistically high values. Furthermore the condensation force dominates in the lower atmosphere with the buoyant force more pronounced only in the upper atmosphere. We note that both the buoyant and condensation forces are vertically directed. But we emphasise that their action in the atmosphere is manifested in the formation of horizontal pressure gradients. This follows from the independent stipulation that the atmosphere is vertically in approximate hydrostatic equilibrium. In Sect. 4 we derive the horizontal pressure gradients associated with the condensation force.

\section{Relevance of the condensation-induced pressure changes for atmospheric processes}

\subsection{Horizontal pressure gradients associated with vapor condensation}

We have shown that condensation of water vapor produces a drop of air pressure in the lower atmosphere up to an altitude of a few kilometers, Fig. 1c, in a moist saturated hydrostatically adjusted column. In the dynamic atmospheric context the vapor condenses and latent heat is released during the ascent of moist air. The vertical displacement of air is inevitably accompanied by its horizontal displacement. This translates much of the condensation-induced pressure difference to a horizontal pressure gradient. Indeed, as the upwelling air loses its water vapor, the surface pressure diminishes via hydrostatic adjustment producing a surface gradient of total air pressure between the areas of ascent and descent. The resulting horizontal pressure gradient is proportional to the the ratio of vertical to horizontal velocity $w / u$ (Makarieva and Gorshkov, 2009b).

We will illustrate this point regarding the magnitude of the resulting atmospheric pressure gradient for the case of a stationary flow where the air moves horizontally along the $\mathrm{x}$ axis and vertically along the z-axis; there is no dependence of the flow on the y coordinate. The stationary continuity equation for the mixture of condensable (vapor) and noncondensable (dry air) gases can be written as

$$
\frac{\partial\left(N_{\mathrm{d}} u\right)}{\partial x}+\frac{\partial\left(N_{\mathrm{d}} w\right)}{\partial z}=0
$$

$\frac{\partial\left(N_{\mathrm{v}} u\right)}{\partial x}+\frac{\partial\left(N_{\mathrm{v}} w\right)}{\partial z}=S$

$S \equiv w\left(\frac{\partial N_{\mathrm{v}}}{\partial z}-\frac{N_{\mathrm{v}}}{N} \frac{\partial N}{\partial z}\right)=w N \frac{\partial \gamma}{\partial z}, N=N_{\mathrm{v}}+N_{\mathrm{d}}$.

Here $N_{\mathrm{d}}$ and $N_{\mathrm{v}}$ are molar densities of dry air and saturated water vapor, respectively; $\gamma \equiv N_{\mathrm{v}} / N$, see Eq. (2), $S$ (Eq. 34) is the sink term describing the non-conservation of the condensable component (water vapor). Saturated pressure of water vapor depends on temperature alone. Assuming that vapor is saturated at the isothermal surface we have $\partial N_{\mathrm{v}} / \partial x=0$, so $N_{\mathrm{v}}$ only depends on $z$. (This condition necessitates either that there is an influx of water vapor via evaporation from the surface (if the circulation pattern is 
immobile), or that the pressure field moves as vapor is locally depleted. The second case occurs in compact circulation patterns like hurricanes and tornadoes (Makarieva and Gorshkov, 2011; Makarieva et al., 2011).) As the air ascends with vertical velocity $w$, vapor molar density decreases due to condensation and due to the expansion of the gas along the vertical gradient of decreasing pressure. The latter effect equally influences all gases, both condensable and noncondensable. Therefore, the volume-specific rate $S(x, z)$ at which vapor molecules are locally removed from the gaseous phase is equal to $w\left[\partial N_{\mathrm{v}} / \partial z-\left(N_{\mathrm{v}} / N\right) \partial N / \partial z\right]$, see Eqs. (1) and (2). The second term describes the expansion of vapor at a constant mixing ratio which would have occurred if vapor were non-condensable as the other gases. (If vapor did not condense, its density would decrease with height as a constant proportion of the total molar density of moist air as with any other atmospheric gas.) Further clarification of the meaning of (Eq. 34) is provided in Sect. 4.2 below, and in Appendix A which offers additional interpretation, see also (Gorshkov et al., 2012).

The mass of dry air is conserved, Eq. (32). Using this fact, Eq. (34) and $\partial N_{\mathrm{v}} / \partial x=0$ in Eq. (33) one can see that

$N\left(\frac{\partial u}{\partial x}+\frac{\partial w}{\partial z}\right)+w \frac{\partial N}{\partial z}=0$.

Now expressing $\partial N / \partial x=\partial N_{\mathrm{d}} / \partial x+\partial N_{\mathrm{v}} / \partial x$ from Eqs. (32) and (33) with use of Eq. (35) we obtain

$\frac{\partial N}{\partial x}=\frac{w}{u}\left(\frac{\partial N_{\mathrm{v}}}{\partial z}-\frac{N_{\mathrm{v}}}{N} \frac{\partial N}{\partial z}\right)$.

Using the equation of state for moist air $p=N R T$ and water vapor $p_{\mathrm{v}}=N_{\mathrm{v}} R T$ we obtain from Eqs. (36) and (25):

$\frac{\partial p}{\partial x}=\left(\frac{\partial p_{\mathrm{v}}}{\partial z}-\frac{p_{\mathrm{v}}}{p} \frac{\partial p}{\partial z}\right) \frac{w}{u}=-\frac{\gamma p}{h_{\gamma}} \frac{w}{u}$.

Here velocities $w$ and $u$ represent vertical and horizontal (along x-axis) velocities of the ascending air flow, respectively. Scale height $h_{\gamma}$ is defined in Eq. (25). A closely related formula for horizontal pressure gradient can be applied to an axis-symmetric stationary flow with $\partial p / \partial x$ replaced by radial gradient $\partial p / \partial r$ (Makarieva and Gorshkov, 2009b).

Equation (37) shows that the difference between the scale heights $h_{\mathrm{v}}$ and $h$ (Eq. 25) of the vertical pressure distributions for water vapor and moist air leads to the appearance of a horizontal pressure gradient of moist air as a whole (Makarieva and Gorshkov, 2007; Gorshkov et al., 2012). This equation contains the ratio of vertical to horizontal velocity. Estimating this ratio it is possible to evaluate, for a given circulation, what sorts of horizontal pressure gradients are produced by condensation and whether these gradients are large enough to maintain the observed velocities via the positive physical feedback described by Eq. (37).

For example, for Hadley cells at $h_{\gamma}=9 \mathrm{~km}, \gamma=0.03$ and a typical ratio of $w / u \sim 2 \times 10^{-3}$ (Rex, 1958) we obtain from
Eq. (37) a pressure gradient of $0.7 \mathrm{~Pa} \mathrm{~km}^{-1}$. On a distance of $1500 \mathrm{~km}$ such a gradient would correspond to a pressure difference of around $10 \mathrm{hPa}$, which is close to the upper range of the actually observed pressure differences in the region (e.g., Murphree and Van den Dool, 1988, Fig. 1). Similar pressure differences and gradients, also comparable in magnitude to $\delta p_{\mathrm{s}}$ (Eq. 27) and $\partial p / \partial r$ are observed within cyclones, both tropical and extratropical, and persistent atmospheric patterns in the low latitudes (Holland, 1980; Zhou and Lau, 1998; Brümmer et al., 2000; Nicholson, 2000; Simmonds et al., 2008). For example, the mean depth of Arctic cyclones, $5 \mathrm{hPa}$ (Simmonds et al., 2008), is about ten times smaller than the mean depth of a typical tropical cyclone (Holland, 1980). This pattern agrees well with the ClausiusClapeyron dependence of $\delta p_{\mathrm{s}}$, Fig. 1b, which would predict an 8 to 16 -fold decrease with mean oceanic temperature dropping by $30-40^{\circ} \mathrm{C}$. The exact magnitude of the pressure gradient and air velocities will depend on the horizontal size of the circulation pattern, the magnitude of friction and degree of the radial symmetry (Makarieva and Gorshkov, 2009a,b, 2011; Makarieva et al., 2011).

Our estimate of the horizontal pressure gradient in a Hadley cell illustrates that our approach when coupled to fundamental atmospheric parameters, yields horizontal pressure gradients of magnitudes similar to those actually observed in large-scale circulation patterns. If we had obtained a much smaller magnitude from Eq. (34) we could conclude that the impact of the vapor sink is negligible and cannot explain the observations. This did not happen. Rather the result adds credibility to our proposal that the vapor sink is a major cause of atmospheric pressure gradients.

Difficulties in the understanding of atmospheric circulation relate to circumstances where uncertainty over the dynamics of water vapor play a role - even if the nature of that role remains debatable. For example, modern global circulation models do not satisfactorily account for the water cycle of the Amazon River Basin, with the estimated moisture convergence being half the actual amounts estimated from the observed runoff values (Marengo, 2006). We note that climate science offers no quantitative theory of Hadley circulation based on current theories and the effects of differential heating alone (Held and Hou, 1980; Fang and Tung, 1999; Schneider, 2006). Efforts to address this challenge are ongoing but progress is limited (e.g., Lindzen and Hou, 1988; Robinson, 2006; Walker and Schneider, 2005, 2006). In one recent review concerning theories of general circulation the understanding of atmospheric moisture and its influences, particularly, lack of relevant theoretical concepts, were identified as a persistent challenge (Schneider, 2006).

Furthermore, many climate researchers readily acknowledge that the current incomplete understanding of the general circulation precludes a theory-based analysis, from fundamental physical principles, of the role of latitudinal atmospheric mixing in stabilizing the Earth's thermal regime important - this is not a minor and thus neglected detail 
but is central in debates concerning climate sensitivity (e.g., Lindzen and Choi, 2009; Trenberth et al., 2010). It would seem to many that new ideas are needed. If these ideas were obvious, and followed directly from current paradigms, they would have already been identified and accepted - thus we should not be surprised that the new ideas we all seek may challenge conventional perspectives. We conclude that our approaches are a promising new avenue for further examination.

\subsection{Condensation rate and hydrostatic equilibrium}

Let us dwell in greater detail on the physical meaning of Eq. (34) that specifies condensation rate in a unit volume. The second term in brackets, $\left(N_{\mathrm{v}} / N\right) \partial N / \partial z$, describes how the molar density of vapor would change during adiabatic ascent if the water vapor were non-condensable and there would be no condensation in the column. This reference term is needed to discriminate the density change caused by condensation from the density change due to gravitational expansion. As we presume that moist air as a whole is in hydrostatic equilibrium, see Eq. (20), it is total molar density $N$ that must be used as such a reference. Indeed, total molar density remains in hydrostatic equilibrium in the absence of condensation as well as in its presence. In the limit $N_{\mathrm{v}} \rightarrow N$ Eq. (34) gives a physically meaningful result, $S=0$. Indeed, when atmosphere consists of water vapor only and is in hydrostatic equilibrium, no condensation takes place. Condensation occurs only when water vapor distribution is non-equilibrium.

When condensation is absent, dry air is in hydrostatic equilibrium. But when water vapor condenses and its distribution is compressed several-fold compared to the hydrostatic distribution, the dry air must be "stretched" compared to its hydrostatic distribution. Only in this case, when the nonequilibrium deficit of vapor in the upper atmosphere is compensated by the non-equilibrium excess of dry air, the moist air as a whole will remain in equilibrium. The distribution of $N_{\mathrm{d}}$ is non-equilibrium and cannot be used instead of $N$ in the reference term in Eq. (34).

The horizontal pressure gradient produced by condensation is therefore a direct consequence of hydrostatic adjustment. The air expands upwards to compensate for vapor deficit, thus its pressure at the surface diminishes in the region of ascent. If no hydrostatic adjustment took place, the dry air would remain in hydrostatic equilibrium (while moist air as a whole would not). In this case dry air molar density $N_{\mathrm{d}}$ could be used in the reference term in Eq. (34). Putting $N_{\mathrm{d}}$ instead of $N$ in Eq. (34), i.e., replacing $S$ by $S_{\mathrm{d}} \equiv \partial N_{\mathrm{v}} / \partial z-\left(N_{\mathrm{v}} / N_{\mathrm{d}}\right) \partial N_{\mathrm{d}} / \partial z$ in Eq. (33), and performing all the derivations in Sect. 4.1 , one obtains $\partial p / \partial x=0$. This result is obvious: in the absence of hydrostatic adjustment, the dry air distribution is not affected by condensation and remains in equilibrium both in horizontal and vertical dimensions. The non-equilibrium gradient of total air pressure re- mains located in the vertical dimension and is not translated onto horizontal dimension. Such a situation could take place in an atmosphere that would be much higher than it is wide. In the real atmosphere which is effectively very thin, most part of the non-equilibrium pressure gradient is transferred to the horizontal plane via rapid hydrostatic adjustment. Note that $S_{\mathrm{d}} \equiv S /(1-\gamma)$ and $S \equiv S_{\mathrm{d}} /\left(1+\gamma_{\mathrm{d}}\right), \gamma_{\mathrm{d}} \equiv N_{\mathrm{v}} / N_{\mathrm{d}}$. The expressions for condensation rates in situations with or without hydrostatic adjustment differ, respectively, by the absence or presence of the multiplier $1 /(1-\gamma)$ in Eq. (34).

We emphasize that whether the hydrostatic adjustment takes place or not, the disequilibrium gradient of total air pressure persists, being located, respectively, either in the horizontal or in the vertical dimension. Note that if $S=S_{\mathrm{d}}$ then $S \equiv S_{\mathrm{d}} \equiv 0$, condensation is absent and atmospheric pressure is in equilibrium in all directions (see Appendix A).

When asking for feedback on earlier versions of this text several readers assumed that Eq. (34) for condensation rate is an approximate form of the exact expression (Eq. 33). Here we address this misunderstanding, see also Appendix A for more details. Equation (33) represents a general continuity (mass balance) equation for water vapor. It does not contain any information about condensation - indeed, it is equally valid for condensation $S<0$, evaporation $S>0$ or absence of phase transitions altogether, $S=0$. Also, it is equally valid for any dependence of $S$ on spatial coordinates, velocities, temperature, pressure or any other variables. In other words, the continuity equation universally applies to all circulation events. In the meantime, our task here is to study only those circulation patterns that are induced by condensation associated with adiabatic ascent. To do so, we need to specify term $S$ in Eq. (33) so we can use this equation for the determination of condensation-induced pressure gradients. This is done by means of Eq. (34), which says that: (1) in the considered volume the only source of phase transitions is condensation; (2) this condensation is caused by the adiabatic ascent of moist saturated air (no condensation occurs if the air moves horizontally because of isothermal surface) and (3) that the moist saturated air is in hydrostatic equilibrium. We stress that none of these specific assumptions are contained in the universal continuity equation (Eq. 33). (In contrast to the generally applicable Eq. (33), Eq. (34) would not be valid, for example, for the case of adiabatic descent, or for a horizontal motion along a non-isothermal surface.) We emphasize that $S$ (Eq. 34) is based on specific physical considerations, not on formal mathematical analogies.

\subsection{Regarding previous oversight of the effect}

For many readers a major barrier to acceptance of our propositions may be to understand how such a fundamental physical mechanism has been overlooked until now. Why has this theory come to light only now in what is widely regarded as a mature field? We can offer a few thoughts based on our readings and discussions with colleagues. 
The condensation-induced pressure gradients that we have been examining are associated with density gradients that have been conventionally considered as minor and thus ignored in the continuity equation (e.g., Sabato, 2008). For example, a typical $\Delta p=50 \mathrm{hPa}$ pressure difference observed along the horizontally isothermal surface between the outer environment and the hurricane center (e.g., Holland, 1980) is associated with a density difference of only around $5 \%$. This density difference can be safely neglected when estimating the resulting air velocity $u$ from the known pressure differences $\Delta p$. Here the basic scale relation is given by Bernoulli's equation, $\rho u^{2} / 2=\Delta p$. The point is that a $5 \%$ change in $\rho$ does not significantly impact the magnitude of the estimated air velocity at a given $\Delta p$. But, as we have shown in the previous section, for the determination of the pressure gradient (Eq. 37) the density difference and gradient (Eq. 36) are key.

Considering the equation of state (Eq. 5) for the horizontally isothermal surface we have $p=C \rho$, where $C \equiv R T / M=$ const. Irrespective of why the considered pressure difference arises, from Bernoulli's equation we know that $u^{2}=2 \Delta p / \rho=2 C \Delta \rho / \rho, \Delta \rho=\rho_{0}-\rho$. Thus, if one puts $\Delta \rho / \rho=\Delta p / p$ equal to zero, no velocity forms and there is no circulation. Indeed, we have $u^{2}=2 \Delta p / \rho=2 C \Delta \rho / \rho=2 C\left(\Delta \rho / \rho_{0}\right)\left(1+\Delta \rho / \rho_{0}+\ldots\right)$. As one can see, discarding $\Delta \rho$ compared to $\rho$ does indeed correspond to discarding the higher order term of the smallness parameter $\Delta \rho / \rho$. But with respect to the pressure gradient, the main effect is proportional to the smallness parameter $\Delta \rho / \rho_{0}$ itself. If the latter is assumed to be zero, the effect is overlooked. We suggest that this dual aspect of the magnitude of condensation-related density changes has not been recognized and this has contributed to the neglect of condensationassociated pressure gradients in the Earth's atmosphere.

Furthermore, the consideration of air flows associated with phase transitions of water vapor has been conventionally reduced to the consideration of the net fluxes of matter ignoring the associated pressure gradients. Suppose we have a linear circulation pattern divided into the ascending and descending parts, with similar evaporation rates $E\left(\mathrm{~kg} \mathrm{H}_{2} \mathrm{O} \mathrm{m}^{-2} \mathrm{~s}^{-1}\right)$ in both regions. In the region of ascent the water vapor precipitates at a rate $P$. This creates a mass sink $E-P$, which has to be balanced by water vapor import from the region of descent. Approximating the two regions as boxes of height $h$, length $l$ and width $d$, the horizontal velocity $u_{t}$ associated with this mass transport can be estimated from the mass balance equation

$l d(P-E)=u_{t} \rho h d, u_{t}=\frac{(P-E)}{\rho} \frac{l}{h}$.

Equation (38) says that the depletion of air mass in the region of ascent at a total rate of $l d(P-E)$ is compensated for by the horizontal air influx from the region of descent that goes with velocity $u_{t}$ via vertical cross-section of area $h d$. For typical values in the trop- ics with $P-E \sim 5 \mathrm{~mm} \mathrm{~d}^{-1}=5.8 \times 10^{-5} \mathrm{~kg} \mathrm{H}_{2} \mathrm{O} \mathrm{m}^{-2} \mathrm{~s}^{-1}$ and $l / h \sim 2 \times 10^{3}$ we obtain $u_{t} \sim 1 \mathrm{~cm} \mathrm{~s}^{-1}$. For regions where precipitation and evaporation are smaller, the value of $u_{t}$ will be smaller too. For example, Lorenz (1967) estimated $u_{t}$ to be $\sim 0.3 \mathrm{~cm} \mathrm{~s}^{-1}$ for the air flow across latitude $40^{\circ} \mathrm{S}$.

With $\rho \approx \rho_{\mathrm{d}}$ the value of $u_{t}$ can be understood as the massweighted horizontal velocity of the dry air+water vapor mixture, which is the so-called barycentric velocity, see, e.g., (Wacker and Herbert, 2003; Wacker et al., 2006). There is no net flux of dry air between the regions of ascent and descent, but there is a net flux of water vapor from the region of descent to the region of ascent. This leads to the appearance of a non-zero horizontal velocity $u_{t}$ directed towards the region of ascent. Similarly, vertical barycentric velocity at the surface is $w_{t} \approx(E-P) / \rho$ (Wacker and Herbert, 2003), which reflects the fact that there is no net flux of dry air via the Earth's surface, while water vapor is added via evaporation or removed through precipitation. The absolute magnitude of vertical barycentric velocity $w_{t}$ for the calculated tropical means is vanishingly small, $w_{t} \sim+0.05 \mathrm{~mm} \mathrm{~s}^{-1}$.

We speculate that the low magnitude of barycentric velocities has contributed to the judgement that water's phase transitions cannot be a major driver of atmospheric dynamics. However, barycentric velocities should not be confused with the actual air velocities (e.g., Meesters et al., 2009). Unlike the former, the latter cannot be estimated without considering atmospheric pressure gradients (Makarieva and Gorshkov, 2009c). For example, in the absence of friction, the maximum linear velocity $u_{c}$ that could be produced by condensation in a linear circulation pattern in the tropics constitutes

$u_{c}=\sqrt{2 \Delta p / \rho} \sim 40 \mathrm{~m} \mathrm{~s}^{-1} \gg u_{t}$.

Here $\Delta p$ was taken equal to $10 \mathrm{hPa}$ as estimated from Eq. (37) for Hadley cell in Sect. 4.1. As one can see, $u_{c}$ (Eq. 39) is much greater than $u_{t}$ (Eq. 38). As some part of potential energy associated with the condensation-induced pressure gradient is lost to friction (Makarieva and Gorshkov, 2009a), real air velocities observed in large-scale circulation are an order of magnitude smaller than $u_{c}$, but still nearly three orders of magnitude greater than $u_{t}$.

\subsection{The dynamic efficiency of the atmosphere}

We will now present another line of evidence for the importance of condensation-induced dynamics: we shall show that it offers an improved understanding of the efficiency with which the Earth's atmosphere can convert solar energy into kinetic energy of air circulation. While the Earth on average absorbs about $I \approx 2.4 \times 0^{2} \mathrm{~W} \mathrm{~m}^{-2}$ of solar radiation (Raval and Ramanathan, 1989), only a minor part $\eta \sim 10^{-2}$ of this energy is converted to the kinetic power of atmospheric and oceanic movement. Lorenz (1967, p. 97) notes, "the determination and explanation of efficiency $\eta$ constitute the fundamental observational and theoretical problems of 
atmospheric energetics". Here the condensation-induced dynamics yields a relationship that is quantitative in nature and can be estimated directly from fundamental atmospheric parameters.

A pressure gradient is associated with a store of potential energy. The physical dimension of pressure gradient coincides with the dimension of force per unit air volume, i.e. $1 \mathrm{~Pa} \mathrm{~m}^{-1}=1 \mathrm{~N} \mathrm{~m}^{-3}$. When an air parcel moves along the pressure gradient, the potential energy of the pressure field is converted to the kinetic energy. The dimension of pressure is identical to the dimension of energy density: $1 \mathrm{~Pa}=1 \mathrm{~N} \mathrm{~m}^{-2}=1 \mathrm{~J} \mathrm{~m}^{-3}$. As the moist air in the lower part of the atmospheric column rises to height $h_{\gamma}$ where most part of its water vapor condenses, the potential energy released amounts to approximately $\delta p_{\mathrm{s}}$ (Eq. 27). The potential energy released $\pi_{\mathrm{v}}$ per unit mass of water vapor condensed, dimension $\mathrm{J}\left(\mathrm{kg} \mathrm{H}_{2} \mathrm{O}\right)^{-1}$, thus becomes

$\pi_{\mathrm{v}}\left(T_{\mathrm{s}}\right)=\frac{\delta p_{\mathrm{s}}}{\rho_{\mathrm{v}}}=\frac{R T_{\mathrm{s}}}{M_{\mathrm{v}}}\left(1-\frac{M_{\mathrm{v}} g T_{\mathrm{s}}}{L \Gamma_{\mathrm{s}}}\right)$.

The global mean precipitation rate is $P \sim 10^{3} \mathrm{~kg} \mathrm{H}_{2} \mathrm{O} \mathrm{m}^{-2} \mathrm{yr}^{-1}$ (L'vovitch, 1979), global mean surface temperature is $T_{\mathrm{S}}=288 \mathrm{~K}$ and the observed mean tropospheric lapse rate $\Gamma_{\mathrm{o}}=6.5 \mathrm{~K} \mathrm{~km}^{-1}$ (Glickman, 2000). Using these values and putting $\Gamma_{\mathrm{o}}$ instead of the moist adiabatic lapse rate $\Gamma_{\mathrm{s}}$ in Eq. (40), we can estimate the global mean rate $\Pi_{\mathrm{v}}=P \pi_{\mathrm{v}}$ at which the condensation-related potential energy is available for conversion into kinetic energy. At the same time we also estimate the efficiency $\eta=\Pi_{\mathrm{v}} / I$ of atmospheric circulation that can be generated by solar energy via the condensation-induced pressure gradients:

$\Pi_{\mathrm{v}}=P \pi_{\mathrm{v}} \sim 3.5 \mathrm{Wm}^{-2}, \eta \sim 0.015$.

Thus, the proposed approach not only clarifies the dynamics of solar energy conversion to the kinetic power of air movement (solar power spent on evaporation $\rightarrow$ condensationrelated release of potential power $\rightarrow$ kinetic power generation), but it does so in a quantiatively tractable manner, explaining the magnitude of the dissipative power associated with maintaining the kinetic energy of the Earth's atmosphere.

Our estimate of atmospheric efficiency differs fundamentally from a thermodynamic approach based on calculating the entropy budgets under the assumption that the atmosphere works as a heat engine, e.g. Pauluis et al. (2000); Pauluis and Held (2002a,b), see also Makarieva et al. (2010). The principal limitation of the entropy-budget approach is that while the upper bounds on the amount of work that could be produced are clarified, there is no indication regarding the degree to which such work is actually performed. In other words, the presence of an atmospheric temperature gradient is insufficient to guarantee that mechanical work is produced. In contrast, our estimate (Eq. 41) is based on an explicit calculation of mechanical work derived from a defined atmospheric pressure gradient. It is, to our knowledge, the only available estimate of efficiency $\eta$ made from the basic physical parameters that characterize the atmosphere.

\subsection{Evaporation and condensation}

While condensation releases the potential energy of atmospheric water vapor, evaporation, conversely, replenishes it. Here we briefly dwell on some salient differences between evaporation and condensation to complete our picture regarding how the phase transitions of water vapor generate pressure gradients.

Evaporation requires an input of energy to overcome the intermolecular forces of attraction in the liquid water to free the water molecule to the gaseous phase, as well as to compress the air. That is, work is performed against local atmospheric pressure to make space for vapor molecules that are being added to the atmosphere via evaporation. This work, associated with evaporation, is the source of potential energy for the condensation-induced air circulation. Upon condensation, two distinct forms of potential energy arise. One is associated with the potential energy of raised liquid drops this potential energy dissipates to friction as the drops fall. The second form of potential energy is associated with the formation of a non-equilibrium pressure gradient, as the removal of vapor from the gas phase creates a pressure shortage of moist air aloft. This pressure gradient produces air movement. In the stationary case total frictional dissipation in the resulting circulation is balanced by the fraction of solar power spent on the work associated with evaporation.

Evaporation is, fundamentally, a surface-specific process because it represents a flux of water molecules via the surface of liquid. In contrast, condensation is a volume-specific process that affects vapor molecules distributed in a certain volume. The balance between condensation and evaporation demands that to compensate for the amount of moisture condensed in a certain volume vapor must be transported to that local volume via its borders. Adding more gas to a gas volume where condensation has occurred is associated with compression of the gas in the volume and, hence, with performing work on the gas.

In the stationary case, as long there is a supply of energy and the relative humidity is less than unity, evaporation from the planetary surface is adding water vapor to the atmospheric column without changing its temperature. The rate of evaporation is affected by turbulent mixing and is usually related to the horizontal wind speed at the surface. The global mean power of evaporation cannot exceed the power of solar radiation.

The primary cause of condensation is the cooling of air masses as the moist air ascends and its temperature drops. Provided there is enough water vapor in the ascending air, at a local and short-term scale, condensation is not governed by solar power but by stored energy and can occur at an arbitrarily high rate dictated by the vertical velocity of the ascending flow, see Eq. (34). 
Any circulation pattern includes areas of lower pressure where air ascends, as well as higher pressure areas where it descends. Condensation rates are non-uniform across these areas - being greater in areas of ascent. Importantly, in such areas of ascent condensation involves water vapor that is locally evaporated along with often substantial amounts of additional water vapor transported from elsewhere. Therefore, the mean rate of condensation in the ascending region of any circulation pattern is always higher than the local rate of evaporation. This inherent spatial non-uniformity of the condensation process determines horizontal pressure gradients.

Consider a large-scale stationary circulation where the regions of ascent and descent are of comparable size. A relevant example would be the annually averaged circulation between the Amazon River Basin (the area of ascent) and the region of Atlantic ocean where the air returns from the Amazon to descend depleted of moisture. Assuming that the relative humidity at the surface, horizontal wind speed and solar power are approximately the same in the two regions, mean evaporation rates should be roughly similar as well (i.e., coincide at least in the order of magnitude). However, the condensation (and precipitation) rates in the two regions will be consistently different. In accordance with the picture outlined above, the average precipitation rate $P_{\mathrm{a}}$ in the area of ascent should be approximately double the average value of regional evaporation rate $E_{\mathrm{a}}$. The pressure drop caused by condensation cannot be compensated by local evaporation so as to produce a net zero effect on air pressure. This is because in the region of ascent both the local water vapor evaporated from the forest canopy of the Amazon forest at a rate $E_{\mathrm{a}} \sim E_{\mathrm{d}}$ as well as imported water vapor evaporated from the ocean surface at a rate $E_{\mathrm{d}}$ precipitate, $P_{\mathrm{a}}=E_{\mathrm{d}}+E_{\mathrm{a}}$. This is confirmed by observations: precipitation in the Amazon river basin is approximately double the regional evaporation, $P_{\mathrm{a}} \approx 2 E_{\mathrm{a}}$ (Marengo, 2004). The difference between regional rates of precipitation and evaporation on land, $R=P_{\mathrm{a}}-E_{\mathrm{a}} \sim E_{\mathrm{a}}$, is equal to regional runoff - aerial or liquid. In the region of descent the runoff thus defined is negative and corresponds to the flux of water vapor that is exported away from the region with the air flow. Where $R$ is positive, it represents the flux of imported atmospheric water vapor and the equal flux of liquid water that leaves the region of ascent to the ocean.

The fact that the climatological means of evaporation and precipitation are seldom observed to be equal has been recognized in the literature (e.g., Wacker and Herbert, 2003), as has the fact that local mean precipitation values are consistently larger than those for evaporation (e.g., Trenberth et al., 2003).

The inherent spatial non-uniformity of the condensation process explains why it is condensation that principally determines the pressure gradients associated with water vapor. So, while evaporation is adding vapor to the atmosphere and thus increasing local air pressure, while condensation in contrast decreases it, the evaporation process is significantly more even and uniform spatially than is condensation. Roughly speaking, in the considered example evaporation increases pressure near equally in the regions of ascent and descent, while condensation decreases pressure only in the region of ascent. Moreover, as discussed above, the rate at which the air pressure is decreased by condensation in the region of ascent is always higher than the rate at which local evaporation would increase air pressure. The difference between the two rates is particularly marked in heavily precipitating systems like hurricanes, where precipitation rates associated with strong updrafts can exceed local evaporation rates by more than an order of magnitude (e.g., Trenberth and Fasullo, 2007).

We have so far discussed the magnitude of pressure gradients that are produced and maintained by condensation in the regions where the moist air ascends. This analysis is applicable to observed condensation processes that occur on different spatial scales, as we illustrated on the example of Hadley cell. We emphasize that to determine where the ascending air flow and condensation predominantly occur is a separate physical problem. For example, why the updrafts are located over the Amazon and the downdrafts are located over the Atlantic ocean and not vice versa. Here regional evaporation patterns play a crucial role. In Sect. 4.1 we have shown that constant relative humidity associated with surface evaporation, which ensures that $\partial N_{\mathrm{v}} / \partial x=0$, is necessary for the condensation to take place. Using the definition of $\gamma$ (Eq. 2) Eq. (37) can be re-written as follows:

$\frac{\partial \ln \gamma}{\partial x}=-\frac{w}{u} \frac{\partial \gamma}{\partial z}$.

This equation shows that the decrease of $\gamma$ with height and, hence, condensation is only possible when $\gamma$ grows in the horizontal direction, $\partial \ln \gamma / \partial x>0$. Indeed, surface pressure is lower in the region of ascent. As the air moves towards the region of low pressure, it expands. In the absence of evaporation, this expansion would make the water vapor contained in the converging air unsaturated. Condensation at a given height would stop.

Evaporation adds water vapor to the moving air to keep water vapor saturated and sustain condensation. The higher the rate of evaporation, the larger the ratio $w / u$ at a given $\partial \gamma / \partial z$ and, hence, the larger the pressure gradient (Eq. 37) that can be maintained between the regions of ascent and descent. A small, but persistent difference in mean evaporation $\Delta E<E$ between two adjacent regions, determines the predominant direction of the air flow. This explains the role of the high leaf area index of the natural forests in keeping evaporation higher than evaporation from the open water surface of the ocean, for the forests to become the regions of low pressure to draw moist air from the oceans and not vice versa (Makarieva and Gorshkov, 2007, 2010; Makarieva et al., 2013). Where the surface is homogeneous with respect to evaporation (e.g., the oceanic surface), the 
spatial and temporal localization of condensation events is likely to fluctuate in a random fashion.

\section{Discussion: condensation dynamics versus differential heating in the generation of atmospheric circulation}

In Sect. 2 we argued that condensation cannot occur adiabatically at constant volume but is always accompanied by a pressure drop in the local air volume where it occurs. We concluded that the statement that "the pressure drop by adiabatic condensation is overcompensated by latent heat induced pressure rise of the air" (Pöschl, 2009, p. S12437) was not correct. In Sect. 3 we quantified the pressure change produced by condensation as dependent on altitude in a column in hydrostatic balance, to show that in such a column the pressure drops upon condensation everywhere in the lower atmosphere up to several kilometers altitude, Fig. 1c. The estimated pressure drop at the surface increases exponentially with growing temperature and amounts to over $20 \mathrm{hPa}$ at $300 \mathrm{~K}$, Fig. $1 \mathrm{~b}$.

In Sect. 4 we discussed the implications of the condensation-induced pressure drop for atmospheric dynamics. We calculated the horizontal pressure gradients produced by condensation and the efficiency of the atmosphere as a dynamic machine driven by condensation. Our aim throughout has been to persuade the reader that these implications are significant in numerical terms and deserve a serious discussion and further analysis. We will conclude by discussing condensation in contrast to differential heating, the latter conventionally considered the major driver dominating atmospheric dynamics.

Atmospheric circulation is only maintained if, in agreement with the energy conservation law, there is a pressure gradient to accelerate the air masses and sustain the existing kinetic energy of air motion against dissipative losses. For centuries, starting from the works of Hadley and his predecessors, the air pressure gradient has been qualitatively associated with the differential heating of the Earth's surface and the Archimedes force (buoyancy) which makes the warm and light air rise, and the cold and heavy air sink (e.g., Gill, 1982, p. 24). This idea can be illustrated by Fig. 1c, where the warmer atmospheric column appears to have higher air pressure at some heights than the colder column. In the conventional paradigm, this is expected to cause air divergence aloft away from the warmer column, which, in its turn, will cause a drop of air pressure at the surface and the resulting surface flow from the cold to the warm areas. Despite the physics of this differential heating effect being straightforward in qualitative terms, the quantitative problem of predicting observed wind velocities from the fundamental physical parameters has posed enduring difficulties. Slightly more than a decade before the first significant efforts in computer climate modelling, Brunt (1944) as cited by Lewis (1998) wrote:
"It has been pointed out by many writers that it is impossible to derive a theory of the general circulation based on the known value of the solar constant, the constitution of the atmosphere, and the distribution of land and sea ... It is only possible to begin by assuming the known temperature distribution, then deriving the corresponding pressure distribution, and finally the corresponding wind circulation".

Brunt's difficulty relates to the realization that pressure differences associated with atmospheric temperature gradients cannot be fully transformed into kinetic energy. Some energy is lost to thermal conductivity without generating mechanical work. This fraction could not be easily estimated by theory in his era - and thus it has remained to the present. The development of computers and appearance of rich satellite observations have facilitated empirical parameterizations to replicate circulation in numerical models. However, while these models provide reasonable replication of the quantitative features of the general circulation they do not constitute a quantitative physical proof that the the observed circulation is driven by pressure gradients associated with differential heating. As Lorenz (1967, p. 48) emphasized, although "it is sometimes possible to evaluate the long-term influence of each process affecting some feature of the circulation by recourse to the observational data", such knowledge "will not by itself constitute an explanation of the circulation, since it will not reveal why each process assumes the value which it does".

In comparison to temperature-associated pressure difference, the pressure difference associated with water vapor removal from the gas phase can develop over a surface of uniform temperature. In addition, this pressure difference is physically anchored to the lower atmosphere. Unlike the temperature-related pressure difference, it does not demand the existence of some downward transport of the pressure gradient from the upper to the lower atmosphere (i.e., the divergence aloft from the warmer to the colder column as discussed above) to explain the appearance of low altitude pressure gradients and the generation of surface winds.

Furthermore, as the condensation-related pressure difference $\delta p_{\mathrm{s}}$ is not associated with a temperature difference, the potential energy stored in the pressure gradient can be nearly fully converted to the kinetic energy of air masses in the lower atmosphere without losses to heat conductivity. This fundamental difference between the two mechanisms of pressure fall generation can be traced in hurricanes. Within the hurricane there is a marked pressure gradient at the surface. This difference is quantitatively accountable by the condensation process (Makarieva and Gorshkov, 2009b, 2011). In the meantime, the possible temperature difference in the upper atmosphere that might have been caused by the difference in moist versus dry lapse rates between the regions of ascent and descent is cancelled by the strong horizontal 
mixing (Montgomery et al., 2006). Above approximately $1.5 \mathrm{~km}$ the atmosphere within and outside the hurricane is approximately isothermal in the horizontal direction (Montgomery et al., 2006, Fig. 4), see also Knaff et al. (2000). Therefore, while the temperature-associated pressure difference above height $z_{c}$, Fig. 1c, is not realized in the atmosphere, the condensation-associated pressure difference below height $z_{c}$ apparently is.

Some hints on the relative strengths of the circulation driven by differential heating compared to condensationinduced circulation can be gained from evaluating wind velocities in those real processes that develop in the lower atmosphere without condensation. These are represented by dry (precipitation-free) breezes (such as diurnal wind patterns driven by the differential heating of land versus sea surfaces) and dust devils. While both demand very large temperature gradients (vertical or horizontal) to arise as compared to the global mean values, both circulation types are of comparatively low intensity and have negligible significance to the global circulation. For example, dust devils do not involve precipitation and are typically characterized by wind velocities of several meters per second (Sinclair, 1973). The other type of similarly compact rotating vortexes - tornadoes - that are always accompanied by phase transitions of water - develop wind velocities that are at least an order of magnitude higher (Wurman et al., 1996). More refined analyses of Hadley circulation (Held and Hou, 1980) point towards the same conclusion: theoretically described Hadley cells driven by differential heating appear to be one order of magnitude weaker than the observed circulation (Held and Hou, 1980; Schneider, 2006), see also Caballero et al. (2008). While the theoretical description of the general atmospheric circulation remains unresolved, condensation-induced dynamics offers a possible solution (as shown in Sect. 4.1).

Our approach and theory have other significant implications. Some have been discussed in previous papers, for example with regard to the development of hurricanes (Makarieva and Gorshkov, 2009a,b) and the significance of vegetation and terrestrial evaporation fluxes in determining large scale continental weather patterns (Makarieva et al., 2006, 2009; Makarieva and Gorshkov, 2007; Sheil and Murdiyarso, 2009). Recently accumulated evidence directly documents air flows induced by the phase transitions of water vapor (Chikoore and Jury, 2010). Other implications are likely to be important in predicting the global and local nature of climate change - a subject of considerable concern and debate at the present time (Pielke et al., 2009; Schiermeier, 2010).

In summary, although the formation of air pressure gradients via condensation has not received adequate theoretical attention in climatological and meteorological sciences, here we have argued that this lack of attention has been undeserved. Condensation-induced dynamics emerges as a new field of investigations that can significantly enrich our understanding of atmospheric processes and climate change. We very much hope that our present account will provide a spur for further investigations both theoretical and empirical into these important, but as yet imperfectly characterized, phenomena.

\section{Appendix A}

\section{On the physical meaning of Eq. (34) for condensation rate}

Equation (34) expresses condensation rate as the difference between (a) the total change of vapor density with height and (b) the density change caused by adiabatic expansion. Here we explore the physical meaning of this expression from a different perspective. We shall show that Eq. (34) follows directly from the condition that the vertical distribution of moist air remains in equilibrium under the assumption that condensation rate $S$ is linear over the amount of vapor (i.e., condensable gas) in the atmosphere.

\section{A1 Linearity of condensation rate over the molar density $N_{v}$ of water vapor}

The linearity assumption is justified by the particular physical nature and stoichiometry of condensation, with gas turning to liquid: condensation is a first-order reaction over saturated molar density $N_{\mathrm{v}}$ of the condensing gas. This can be experimentally tested by considering condensation of water with different isotopic composition (e.g., Fluckiger and Rossi, 2003). (Note, for example, that the reverse process (evaporation) is a zero-order reaction over $N_{\mathrm{v}}$.)

The rate of first-order reactions is directly proportional to the molar density of the reagent, with the proportionality constant having the dimension of inverse time: $S=C N_{\mathrm{v}}$, where $C$ (dimension $\mathrm{s}^{-1}$ ) is in the general case independent of $N_{\mathrm{v}}$. In chemical kinetics $C$ depends on temperature and the molecular properties of the reagent as follows from the law of mass action. Since the saturated concentration $N_{\mathrm{v}}$ of condensable gas depends on temperature as dictated by the Clausius-Clapeyron law, we can ask what the proportionality coefficient $C$ physically means in this case. Different substances have different partial pressures of saturated vapor at any given temperature - this is controlled by the vaporization constant $L$ and the molecular properties of the substance. Note too that for any given substance (like water) the saturated concentration depends on various additional parameters including the curvature of the the liquid surface and availability of condensation nuclei. Therefore, a range of saturated concentrations is possible at any given temperature. This allows one to consider $C$ and $N_{\mathrm{v}}$ as independent variables in the space of all possible combinations of $C$ and $N_{\mathrm{v}}$.

\section{A2 The equilibrium}

The notions of equilibrium and deviation from it are key to determining the rate of any reaction. For example, in the case 
of evaporation the deviation from equilibrium is measured by the water vapor deficit: the deviation of relative humidity from the (equilibrium) unity value. Atmospheric condensation is peculiar in being physically associated with air movement in a particular direction - water vapor condenses as the air moves vertically towards a lower temperature.

Here, in the context of this derivation, by invoking the concept of equilibrium we mean the vertical distribution that the water vapor would locally take in the absence of condensation, all other conditions being equal. Let us denote the inverse scale height of such an equilibrium distribution for $k_{\mathrm{E}}$. Condensation rate $S$ is then proportional to the first order deviation $k_{\mathrm{v}}$ of the observed vertical distribution of water vapor from the equilibrium:

$k_{\mathrm{v}}=-\frac{1}{N_{\mathrm{v}}} \frac{\partial N_{\mathrm{v}}}{\partial z}-k_{\mathrm{E}}$

The physics of Eq. (A1) consists of the fact that the character of the considered equilibrium distribution is not affected by condensation. For example, for the case of hydrostatic equilibrium any gas having molar mass $M$, temperature $T$ and finding itself on a planet with acceleration of gravity $g$ in the presence of a temperature gradient $\partial T / \partial z$ will have a distribution of its molar density following $-\partial N / \partial z=k_{\mathrm{E}} N$, where $k_{\mathrm{E}}=M g / R T+(1 / T) \partial T / \partial z$. (But note that Eq. (A1) can also be applied to describe physical equilibria of a different nature. For example, in a vertically isothermal atmosphere in the absence of gravity $k_{\mathrm{E}}=0$.)

Such a formulation (proportionality of condensation rate to $k_{\mathrm{v}}$ ) presumes that the deviation $k_{\mathrm{v}}$ of the vertical distribution of water vapor from equilibrium is due to condensation alone. (This premise is empirically testable: where condensation is absent, the vertical water vapor distribution should have the same scale height as the non-condensable gases and moist air as a whole.) This removes the need to consider $N_{\mathrm{v}}$ as the saturated vapor concentration. When $k_{\mathrm{V}}=0$, the condensation rate is zero independent of whether water vapor is saturated or not. When $k_{\mathrm{v}} \neq 0, N_{\mathrm{v}}$ is saturated water vapor by formulation.

\section{A3 Distribution of vapor, dry air and moist air as a whole}

We write the condition that moist air with molar density $N$ is in equilibrium in the vertical dimension as:

$-\frac{1}{N} \frac{\partial N}{\partial z} \equiv k=k_{\mathrm{E}}, N=N_{\mathrm{v}}+N_{\mathrm{d}}$

Condensation causes the distribution of vapor $N_{\mathrm{v}}$ to deviate from the equilibrium distribution. The condition that moist air as a whole nevertheless remains in equilibrium causes dry air $N_{\mathrm{d}}$ to also deviate from the equilibrium - but in the opposite direction to the vapor:

$-\frac{\partial N_{\mathrm{v}}}{\partial z}=\left(k+k_{\mathrm{v}}\right) N_{\mathrm{v}},-\frac{\partial N_{\mathrm{d}}}{\partial z}=\left(k+k_{\mathrm{d}}\right) N_{\mathrm{d}}$, $k_{\mathrm{v}} N_{\mathrm{v}}+k_{\mathrm{d}} N_{\mathrm{d}}=0$,

$k_{\mathrm{v}}=-\frac{1}{N_{\mathrm{v}}} \frac{\partial N_{\mathrm{v}}}{\partial z}-k, k_{\mathrm{d}} \equiv-\frac{1}{N_{\mathrm{d}}} \frac{\partial N_{\mathrm{d}}}{\partial z}-k$.

The value of $k_{\mathrm{v}}$ describes the intensity of the mass sink. In the case of water vapor $k_{\mathrm{v}}>0$ is caused by a steep vertical temperature gradient that causes vapor to condense (Makarieva and Gorshkov, 2007; Gorshkov et al., 2012, Sect. 3). From consideration of the Clausius-Clapeyron law and hydrostatic equilibrium one can see that

$k_{\mathrm{v}}=\frac{L \Gamma}{R T^{2}}-\frac{M g}{R T}$,

where $L$ is molar vaporization constant, $\Gamma \equiv-\partial T / \partial z$ is temperature lapse rate, and $M$ is molar mass of air.

The value of $k_{\mathrm{v}}$ is controlled by temperature lapse rate $\Gamma-$ keeping all other variables constant, changing $\Gamma$ it is possible for $k_{\mathrm{v}}$ to take any value, $-\infty<k_{\mathrm{v}}<\infty$. This validates our assumption that $k_{\mathrm{v}}$ can be kept independent of $N_{\mathrm{v}}$ when investigating the limit behavior $N_{\mathrm{v}} \rightarrow 0$ in Eq. (A10): for any $N_{\mathrm{v}}$ (e.g., set by ambient temperature) any value of $k_{\mathrm{v}}$ can be prescribed by changing $\Gamma$.

\section{A4 The limit behaviour $\partial N_{d} / \partial x \rightarrow 0$}

Using Eqs. (32), (33) and $\partial N_{\mathrm{v}} / \partial x=0$ we obtain (see also Gorshkov et al., 2012):

$u \frac{\partial N_{\mathrm{d}}}{\partial x}=\left(S_{\mathrm{d}}-S\right) \frac{1}{\gamma_{\mathrm{d}}}$,

where

$S_{\mathrm{d}} \equiv w\left(\frac{\partial N_{\mathrm{v}}}{\partial z}-\gamma_{\mathrm{d}} \frac{\partial N_{\mathrm{d}}}{\partial z}\right), \gamma_{\mathrm{d}} \equiv \frac{N_{\mathrm{v}}}{N_{\mathrm{d}}}$.

The magnitude of condensation rate $S$ in Eq. (A7) remains unknown. Note that under terrestrial conditions $1 / \gamma_{\mathrm{d}} \gg 1$.

Putting Eq. (A3) into Eq. (A7) using Eq. (A4) we obtain:

$u \frac{\partial N_{\mathrm{d}}}{\partial x}=-w k_{\mathrm{v}} N_{\mathrm{d}}\left(1+\frac{N_{\mathrm{v}}}{N_{\mathrm{d}}}+\frac{S}{w k_{\mathrm{v}} N_{\mathrm{v}}}\right)$.

Now putting $S=C N_{\mathrm{v}}$ into Eq. (A9) we have

$\frac{\partial N_{\mathrm{d}}}{\partial x}=-w k_{\mathrm{v}} \frac{N_{\mathrm{d}}}{u}\left(1+\frac{N_{\mathrm{v}}}{N_{\mathrm{d}}}+\frac{C}{w k_{\mathrm{v}}}\right)$.

We require that $\partial N_{\mathrm{d}} / \partial x \rightarrow 0$ at $N_{\mathrm{v}} \rightarrow 0$ (no horizontal density gradient in the absence of condensable substance). This condition follows from considering that, aside from condensation, there are no processes in the atmospheric column that would make the air distribution deviate from a static equilibrium. This limit is general and should apply to all conditions, 
including cases where all other variables in Eq. (A10) are independent of $N_{\mathrm{v}}$. From this condition we obtain $C=-w k_{\mathrm{v}}$ and

$S=-w k_{\mathrm{v}} N_{\mathrm{v}}$,

which is Eq. (34). An experiment to test this relationship would be to consider a circulation with given vertical and horizontal velocities $w$ and $u$, set $k_{\mathrm{v}}$ and $N_{\mathrm{d}}$ and change the saturated molar density $N_{\mathrm{v}}$ by either changing the condensable gas or the amount of condensation nuclei in the atmosphere or temperature (see below) or both. One will observe that as the condensable gas disappears from the atmosphere, the horizontal pressure gradients vanish. (It is interesting to note the following. Given that the spatial distribution of $N_{\mathrm{v}}$ is exponential, $N_{\mathrm{v}}(z)=N_{0} \exp \left(-z / h_{N}\right)$, the local condition $N_{\mathrm{v}} \rightarrow 0$ corresponds to complete disappearance of the condensable component from the atmosphere and restoration of equilibrium in the horizontal plane. In comparison, the local condition $k_{\mathrm{v}} \rightarrow 0$ does not presume that condensation is absent everywhere else in the atmosphere (it is plausible that $k_{\mathrm{v}}$ changes stepwise at the point where condensation commences).)

In Eq. (A11) condensation rate $S$ is a linear function of three independent variables: vertical velocity $w$, local amount of vapor $N_{\mathrm{v}}$ and deviation $k_{\mathrm{v}}$ of vapor from the equilibrium distribution $\left(k_{\mathrm{v}}\right.$ can be characterized as the "condensability strength" of atmospheric vapor). Note an interesting relationship: with $S$ given by Eq. (A11) and $\gamma \equiv N_{\mathrm{v}} / N$ we have $S_{\mathrm{d}}-S \equiv S \gamma_{\mathrm{d}} \equiv S_{\mathrm{d}} \gamma$. When $S=S_{\mathrm{d}}$ we have $S \equiv S_{\mathrm{d}} \equiv 0$ : condensation is absent.

\section{A5 Appendix summary}

Equations (32) and (33), taken together, contain the information that it is water vapor and not dry air that undergoes condensation. Equation (34) contains information about the magnitude of deviation from equilibrium that causes condensation. Jointly considered, these facts are sufficient to determine the horizontal pressure gradient produced by the vapor sink.

Note that in Eq. (A7) any small difference of the order of $\gamma_{\mathrm{d}}$ between $S$ and $S_{\mathrm{d}}$ is multiplied by a large magnitude $1 / \gamma_{\mathrm{d}} \gg 1$ and thus has a profound influence on the magnitude of the horizontal gradient $\partial N_{\mathrm{d}} / \partial z$. We emphasize the point we made in Sect. 4.2: if it were dry air to be in equilibrium, i.e. $k_{\mathrm{E}}=-\left(1 / N_{\mathrm{d}}\right) \partial N_{\mathrm{d}} / \partial z$, the same consideration of the same equations would give $\partial N_{\mathrm{d}} / \partial x=0$ instead of $\partial N_{\mathrm{d}} / \partial x=S / u$ as in the case when it is moist air that is in equilibrium. The impact of this physical process on atmospheric dynamics remains unexplored.
Acknowledgements. We thank D. R. Rosenfeld and H. H. G. Savenije for disclosing their names as referees in the ACPD discussion of the work of Makarieva et al. (2008) and D. R. Rosenfeld for providing clarifications regarding the derivation of the estimate of condensation-related pressure change as given by Pöschl (2009, p. S12436). We acknowledge helpful comments of K. E. Trenberth towards a greater clarity of the presentation of our approach. The authors benefited greatly from an exciting discussion of condensation-related dynamics with J. I. Belanger, J. A. Curry, G. M. Lackmann, M. Nicholls, R. A. Pielke, G. A. Schmidt, A. Seimon and R. M. Yablonsky. We thank all people who discussed our work, both in the ACPD discussion and in the blogosphere, in particular, J. Condon, J. A. Curry, L. Liljegren, N. Stokes and A. Watts for hosting the discussions on their blogs. We are grateful to P. Restrepo for his interest and help in finding potential reviewers for our work. AMM acknowledges the essential role of S. McIntyre's blog where she came in contact with J. A. Curry. We sincerely thank our two referees, J. A. Curry and I. Held, for their valuable input. We gratefully acknowledge the commitment and insight of A. Nenes and the entire ACP Executive Committee in handling our contribution. BLL thanks the US National Science Foundation and UC Agricultural Experiment Station for their partial support.

\section{Edited by: A. Nenes}

Editor Comment. The authors have presented an entirely new view of what may be driving dynamics in the atmosphere. This new theory has been subject to considerable criticism which any reader can see in the public review and interactive discussion of the manuscript in ACPD (http://www.atmos-chem-phys-discuss.net/ 10/24015/2010/acpd-10-24015-2010-discussion.html). Normally, the negative reviewer comments would not lead to final acceptance and publication of a manuscript in ACP. After extensive deliberation however, the editor concluded that the revised manuscript still should be published - despite the strong criticism from the esteemed reviewers - to promote continuation of the scientific dialogue on the controversial theory. This is not an endorsement or confirmation of the theory, but rather a call for further development of the arguments presented in the paper that shall lead to conclusive disproof or validation by the scientific community. In addition to the above manuscript-specific comment from the handling editor, the following lines from the ACP executive committee shall provide a general explanation for the exceptional approach taken in this case and the precedent set for potentially similar future cases: (1) The paper is highly controversial, proposing a fundamentally new view that seems to be in contradiction to common textbook knowledge. (2) The majority of reviewers and experts in the field seem to disagree, whereas some colleagues provide support, and the handling editor (and the executive committee) are not convinced that the new view presented in the controversial paper is wrong. (3) The handling editor (and the executive committee) concluded to allow final publication of the manuscript in ACP, in order to facilitate further development of the presented arguments, which may lead to disproof or validation by the scientific community. 


\section{References}

Bolton, D.: The computation of equivalent potential temperature, Mon. Weather Rev., 108, 1046-1053, 1980.

Brunt, D.: Physical and Dynamical Meteorology, 2nd Edn., Cambridge University Press, 428 pp., London, UK, 1944.

Brümmer, B., Thiemann, S., and Kirchgäßner, A.: A cyclone statistics for the Arctic based on European centre re-analysis data, Meteorol. Atmos. Phys., 75, 233-250, 2000.

Caballero, R., Pierrehumbert, R. T., and Mitchell, J. L.: Axisymmetric, nearly inviscid circulations in non-condensing radiativeconvective atmospheres, Q. J. Roy. Meteor. Soc., 134, 12691285, 2008.

Chikoore, H. and Jury, M. R.: Intraseasonal variability of satellitederived rainfall and vegetation over Southern Africa, Earth Interact., 14, 1-26, 2010.

Curry, J. A. and Webster, P. J.: Thermodynamics of Atmospheres and Oceans, Academic Press, San Diego, 471 pp., 1999.

Deluc, J. A.: Einige meteorologische Erscheinungen, zu deren genauern Kenntniss die electrische Säule als Luft-Electroscop führen kann, Gilberts Ann. d. Phys., 41, 162-194, 1812.

Gill, A. E.: Atmosphere-Ocean Dynamics, Academic Press, New York, 1982.

Fang, M. and Tung, K. K.: Time-dependent nonlinear Hadley circulation, J. Atmos. Sci., 56, 1797-1807, 1999.

Fluckiger, B. and Rossi, M. J.: Common precursor mechanism for the heterogeneous reaction of $\mathrm{D}_{2} \mathrm{O}, \mathrm{HCl}, \mathrm{HBr}$, and $\mathrm{HOBr}$ with water ice in the range 170-230 K: Mass accommodation coefficients on ice, J. Phys. Chem. A, 107, 4103-4115, 2003.

Glickman, T. S. (Ed.): Glossary of Meteorology, 2nd Edn., Amer. Meteor. Soc., Boston, 855 pp., 2000.

Gorshkov, V. G., Makarieva, A. M., and Nefiodov, A. V.: Condensation of water vapor in the gravitational field, J. Exp. Theor. Phys., 115, 723-728, 2012.

$\mathrm{Gu}, \mathrm{H}$. and Qian, Z: : A discussion about the role of the water vapor source/sink term in continuity equation of numerical models, Chin. Sci. Bull., 36, 16-21, 1991.

Haynes, P. (Ed.): Interactive comment on "On the validity of representing hurricanes as Carnot heat engine" by A. M. Makarieva et al., Atmos. Chem. Phys. Discuss., 8, S12168-S12178, 2009.

Held, I. M. and Hou, A. Y.: Nonlinear axially symmetric circulations in a nearly inviscid atmosphere, J. Atmos. Sci., 37, 515533,1980

Holland, G. J.: An analytic model of the wind and pressure profiles in hurricanes, Mon. Weather Rev., 108, 1212-1218, 1980.

Kis, A. K. and Straka, J. M.: Nocturnal tornado climatology, Weather Forecast., 25, 545-561, 2010.

Knaff, J. A., Zehr, R. M., Goldberg, M. D., and Kidder, S. Q.: An example of temperature structure differences in two cyclone systems derived from the Advanced Microwave Sounder Unit, Weather Forecast., 15, 476-483, 2000.

L'vovitch, M. I.: World Water Resources and Their Future, American Geological Union, Washington, 1979.

Lackmann, G. M. and Yablonsky, R. M.: The importance of the precipitation mass sink in tropical cyclones and other heavily precipitating systems, J. Atmos. Sci., 61, 1674-1692, 2004.

Lewis, J. M.: Clarifying the dynamics of the general circulation: Phillipss 1956 experiment, B. Am. Meteorol. Soc., 79, 39-60, 1998.
Lindzen, R. S. and Hou, A. Y.: Hadley circulations for zonally averaged heating centered off the equator, J. Atmos. Sci., 45, 24162427, 1988.

Lindzen, R. S. and Choi, Y.-S.: On the determination of climate feedbacks from ERBE data, Geophys. Res. Lett., 36, L16705, doi:10.1029/2009GL039628, 2009.

Lorenz, E. N.: The Nature and Theory of the General Circulation of the Atmosphere, World Meteorological Organization, Geneva, 1967.

Lorenz, E. N.: A history of prevailing ideas about the general circulation of the atmosphere, B. Am. Meteorol. Soc., 64, 730-734, 1983.

Makarieva, A. M. and Gorshkov, V. G.: Biotic pump of atmospheric moisture as driver of the hydrological cycle on land, Hydrol. Earth Syst. Sci., 11, 1013-1033, doi:10.5194/hess-11-10132007, 2007.

Makarieva, A. M. and Gorshkov, V. G.: Condensation-induced dynamic gas fluxes in a mixture of condensable and noncondensable gases, Phys. Lett. A, 373, 2801-2804, 2009a.

Makarieva, A. M. and Gorshkov, V. G.: Condensation-induced kinematics and dynamics of cyclones, hurricanes and tornadoes, Phys. Lett. A, 373, 4201-4205, 2009b.

Makarieva, A. M. and Gorshkov, V. G.: Reply to A. G. C. A. Meesters et al.'s comment on "Biotic pump of atmospheric moisture as driver of the hydrological cycle on land", Hydrol. Earth Syst. Sci., 13, 1307-1311, doi:10.5194/hess-13-13072009, 2009c

Makarieva, A. M. and Gorshkov, V. G.: The biotic pump: Condensation, atmospheric dynamics and climate, Inter. Jour. Water, 5 , 365-385, 2010.

Makarieva, A. M. and Gorshkov, V. G.: Radial profiles of velocity and pressure for condensation-induced hurricanes, Phys. Lett. A., 375, 1053-1058, 2011.

Makarieva, A. M., Gorshkov, V. G., and Li, B.-L.: Conservation of water cycle on land via restoration of natural closed-canopy forests: implications for regional landscape planning, Ecol. Res., 21, 897-906, 2006.

Makarieva, A. M., Gorshkov, V. G., and Li, B.-L.: On the validity of representing hurricanes as Carnot heat engine, Atmos. Chem. Phys. Discuss., 8, 17423-17437, doi:10.5194/acpd8-17423-2008, 2008.

Makarieva, A. M., Gorshkov, V. G., and Li, B.-L.: Precipitation on land versus distance from the ocean: evidence for a forest pump of atmospheric moisture, Ecol. Complex., 6, 302-307, 2009.

Makarieva, A. M., Gorshkov, V. G., Li, B.-L., and Nobre, A. D.: A critique of some modern applications of the Carnot heat engine concept: the dissipative heat engine cannot exist, P. R. Soc. A, 466, 1893-1902, 2010.

Makarieva, A. M., Gorshkov, V. G., and Nefiodov, A. V.: Condensational theory of stationary tornadoes, Phys. Lett. A., 375, 22592261, 2011.

Makarieva, A. M., Gorshkov, V. G., and Li, B.-L.: Revisiting forest impact on atmospheric water vapor transport and precipitation, Theor. Appl. Climatol., 111, 79-96, doi:10.1007/s00704012-0643-9, 2013.

Mapes, B. E.: Water's two height scales: the moist adiabat and the radiative troposphere, Q. J. Roy. Meteor. Soc., 127, 2253-2266, 2001. 
Marengo, J. A.: Interdecadal variability and trends of rainfall across the Amazon basin, Theor. Appl. Climatol., 78, 79-96, 2004.

Marengo, J. A.: On the hydrological cycle of the Amazon basin: A historical review and current state-of-the-art, Rev. Bras. Meteorol., 21, 1-19, 2006.

Meesters, A. G. C. A., Dolman, A. J., and Bruijnzeel, L. A.: Comment on "Biotic pump of atmospheric moisture as driver of the hydrological cycle on land" by A. M. Makarieva and V. G. Gorshkov, Hydrol. Earth Syst. Sci., 11, 1013-1033, 2007, Hydrol. Earth Syst. Sci., 13, 1299-1305, doi:10.5194/hess-13-12992009, 2009.

Montgomery, M. T., Bell, M. M., Aberson, S. D., and Black, M. L.: Hurricane Isabel (2003): New insights into the physics of intense storms. Part I: Mean vortex structure and maximum intensity estimates, B. Am. Meteorol. Soc., 87, 1225-1347, 2006.

Murphree, T. and Van den Dool, H.: Calculating tropical winds from time mean sea level pressure fields, J. Atmos. Sci., 45, 32693282, 1988.

Nicholson, S. E.: The nature of rainfall variability over Africa on time scales of decades to millenia, Global Planet. Change, 26, 137-158, 2000.

Ooyama, K. V.: A dynamic and thermodynamic foundation for modeling the moist atmosphere with parameterized microphysics, J. Atmos. Sci., 58, 2073-2102, 2001.

Pauluis, O. P., Balaji, V., and Held, I. M.: Frictional dissipation in a precipitating atmosphere, J. Atmos. Sci., 57, 989-994, 2000.

Pauluis, O. and Held, I. M.: Entropy budget of an atmosphere in radiative-convective equilibrium. Part I: maximum work and frictional dissipation, J. Atmos. Sci., 59, 125-139, 2002a.

Pauluis, O. and Held, I. M.: Entropy budget of an atmosphere in radiative-convective equilibrium. Part II: latent heat transport and moist processes, J. Atmos. Sci., 59, 125-139, 2002 b.

Pielke Sr., R., Beven, K., Brasseur, G., Calvert, J., Chahine, M., Dickerson, R., Entekhabi, D., Foufoula-Georgiou, E., Gupta, H., Gupta, V., Krajewski, W., Krider, E. P., Lau, W. K. M., McDonnell, J., Rossow, W., Schaake, J., Smith, J., Sorooshian, S., and Wood, E.: Climate change: the need to consider human forcings besides greenhouse gases, Eos, 90, p. 413, 2009.

Pöschl, U.: Interactive comment on "On the validity of representing hurricanes as Carnot heat engine" by A. M. Makarieva et al., Atmos. Chem. Phys. Discuss., 8, S12426-S12447, 2009.

Raval, A. and Ramanathan, V.: Observational determination of the greenhouse effect, Nature, 342, 758-761, 1989.

Rex, D. F.: Vertical atmospheric motions in the equatorial Central Pacific, Geophysica, 6, 479-500, 1958.

Qiu, C.-J., Bao, J.-W., and Xu, Q.: Is the mass sink due to precipitation negligible?, Mon. Weather Rev., 121, 853-857, 1993.

Robinson, W. A.: On the self-maintenance of midlatitude jets, J. Atmos. Sci., 63, 2109-2122, 2006.

Trenberth, K. E., Fasullo, J. T., O’Dell, C., and Wong, T.: Relationships between tropical sea surface temperature and topof-atmosphere radiation, Geophys. Res. Lett., 37, L03702, doi:10.1029/2009GL042314, 2010.

Sabato, J. S.: $\mathrm{CO}_{2}$ condensation in baroclinic eddies on early Mars, J. Atmos. Sci., 65, 1378-1395, 2008.

Schiermeier, Q.: The real holes in climate science, Nature, 463, 284-287, 2010.
Schneider, T.: The general circulation of the atmosphere, Annu. Rev. Earth Planet. Sci., 34, 655-688, 2006.

Schubert, W. H., Hausman, S. A., Garcia, M., Ooyama, K. V., and Kuo, H.-C.: Potential vorticity in a moist atmosphere, J. Atmos. Sci., 58, 3148-3157, 2001.

Sheil, D. and Murdiyarso, D.: How forests attract their rain: an examination of a new hypothesis, Bioscience, 59, 341-347, 2009.

Shusse, Y. and Tsuboki, K.: Dimension characteristics and precipitation efficiency of cumulonimbus clouds in the region far south from the mei-yu front over the eastern Asian continent, Mon. Weather Rev., 134, 1942-1953, 2006.

Simmonds, I., Burke, C., and Keay, K.: Arctic climate change as manifest in cyclone behavior, J. Climate, 21, 5777-5796, 2008.

Sinclair, P. C.: The lower structure of dust devils, J. Atmos. Sci., 30, 1599-1619, 1973.

Snodgrass, E. R., Di Girolamo, L., and Rauber, R. M.: Precipitation characteristics of trade wind clouds during RICO derived from radar, satellite, and aircraft measurements, J. Appl. Meteorol. Clim., 48, 464-483, 2009.

Sui, C.-H., Li, X., and Yang, M.-J.: On the definition of precipitation efficiency, J. Atmos. Sci., 64, 4506-4513, 2007.

Thompson, R. L., Edwards, R., Hart, J. A., Elmore, K. L., and Markowski, P.: Close proximity soundings within supercell environments obtained from the rapid update cycle, Weather Forecast., 18, 1243-1261, 2003.

Trenberth, K. E.: Climate diagnostics from global analyses: conservation of mass in ECMWF analyses, J. Climate, 4, 707-722, 1991.

Trenberth, K. E. and Fasullo, J.: Water and energy budgets of hurricanes and implications for climate change, J. Geophys. Res., 112, D23107, doi:10.1029/2006JD008304, 2007.

Trenberth, K. E., Christy, J. R., and Olson, J. G.: Global atmospheric mass, surface pressure, and water vapor variations, J. Geophys. Res., 92, 14815-14826, 1987.

Trenberth, K. E., Dai, A., Rasmussen, R. M., and Parsons, D. B.: The changing character of precipitation, B. Am. Meteorol. Soc., 84, 1205-1217, 2003.

Van den Dool, H. M. and Saha, S.: Seasonal redistribution and conservation of atmospheric mass in a general circulation model, J. Climate, 6, 22-30, 1993.

Wacker, U. and Herbert, F.: Continuity equations as expressions for local balances of masses in cloudy air, Tellus, 55A, 247-254, 2003.

Wacker, U., Frisius, T., and Herbert, F.: Evaporation and precipitation surface effects in local mass continuity laws of moist air, J. Atmos. Sci., 63, 2642-2652, 2006.

Walker, C. C. and Schneider, T.: Eddy-influences on Hadley circulations: simulations with an idealized GCM, J. Atmos. Sci., 63, 3333-3350, 2005.

Walker, C. C. and Schneider, T.: Response of idealized Hadley circulations to seasonally varying heating, Geophys. Res. Lett., 32, L06813, doi:10.1029/2004GL022304, 2006.

Wurman, J., Straka, J. M., and Rasmussen, E. N.: Fine-scale Doppler radar observations of tornadoes, Science, 272, 17741777, 1996.

Zhou, J. and Lau, K.-M.: Does a monsoon climate exist over South America?, J. Climate, 11, 1020-1040, 1998. 